\title{
A mixed problem of linear elastodynamics
}

\author{
Kazuaki Taira \\ Dedicated to Professor Mitsuru Ikawa on the occasion of his 70th birthday
}

Received: date / Accepted: date

\begin{abstract}
This paper is devoted to a semigroup approach to an initial-boundary value problem of linear elastodynamics in the case where the boundary condition is a regularization of the genuine mixed displacement-traction boundary condition. More precisely, it is a smooth linear combination of displacement and traction boundary conditions, but is not equal to the pure traction boundary condition. Some previous results with mixed displacement-traction boundary condition are due to Inoue and Ito. The crucial point in our semigroup approach is to generalize the classical variational approach to the degenerate case, by using the theory of fractional powers of analytic semigroups.
\end{abstract}

Keywords initial-boundary value problem · linear elastodynamics · displacementtraction boundary condition $\cdot$ contraction group

Mathematics Subject Classification (2010) 74B15 - 47D03 · 35J57

\section{Introduction}

This paper is devoted to a semigroup approach to an initial-boundary value problem of linear elastodynamics in the case where the boundary condition is a regularization of the genuine mixed displacement-traction boundary condition. More precisely, it is a smooth linear combination of displacement and traction boundary conditions, but is not equal to the pure traction boundary condition. Some previous results with mixed displacement-traction boundary condition are due to Inoue [14], [15] and Ito [16]. Many problems in partial differential equations can be formulated in terms of abstract operators acting between suitable spaces of distributions, and these operators are then analyzed by the methods of semigroup theory. The crucial point in our semigroup approach is to generalize the classical

Kazuaki Taira

Institute of Mathematics, University of Tsukuba, Tsukuba 305-8571, Japan

Tel.: +81-29-853-4235

Fax: +81-29-853-6501

E-mail: taira@math.tsukuba.ac.jp 
variational approach to the degenerate case, by using the theory of fractional powers of analytic semigroups. The virtue of this approach is that a given problem is stripped of extraneous data, so that the analytic core of the problem is revealed $([24],[7])$.

Let $\Omega$ be an open, connected subset of Euclidean space $\mathbf{R}^{n}, n \geq 2$, with smooth boundary $\partial \Omega$. We think of the closure $\bar{\Omega}=\Omega \cup \partial \Omega$ of $\Omega$ as representing the volume occupied by an undeformed body; so the set $\mathcal{B}=\bar{\Omega}$ is called the reference configuration. In this paper we study the following initial-boundary value problem of linear elastodynamics (see [17, Chapter 6, Section 6.3]): For given vector functions $\boldsymbol{f}(x)=\left(f_{i}(x)\right), \boldsymbol{u}_{0}(x)=\left(u_{0, i}(x)\right)$ and $\boldsymbol{u}_{1}(x)=\left(u_{1, i}(x)\right)$ defined in $\Omega$, find a vector function $\boldsymbol{u}(x)=\left(u_{i}(x)\right)$ in $\Omega$ such that

$$
\begin{cases}\frac{\partial^{2} \boldsymbol{u}}{\partial t^{2}}-\operatorname{div}(\mathbf{a}(x) \cdot \nabla \boldsymbol{u})=\boldsymbol{f} & \text { in } \Omega \times(0, \infty) \\ \left.\boldsymbol{u}\right|_{t=0}=\boldsymbol{u}_{0} & \text { in } \Omega \\ \left.\frac{\partial \boldsymbol{u}}{\partial t}\right|_{t=0}=\boldsymbol{u}_{1} & \text { in } \Omega \\ \alpha(x)(\mathbf{a}(x) \cdot \boldsymbol{\nabla} \boldsymbol{u} \cdot \mathbf{n})+(1-\alpha(x)) \boldsymbol{u}=\mathbf{0} & \text { on } \partial \Omega \times(0, \infty) .\end{cases}
$$

Here:

(1) $\mathbf{a}(x)=\left(a_{i j \ell m}(x)\right)$ is a smooth elasticity tensor.

(2) $\alpha(x)$ is a smooth real-valued function on $\partial \Omega$ such that $0 \leq \alpha(x) \leq 1$ on $\partial \Omega$.

(3) $\mathbf{n}=\left(n_{i}\right)$ is the outward unit normal to $\partial \Omega$.

It is worth pointing out that, componentwise, the initial-boundary value problem (1.1) can be written in the form

$$
\begin{cases}\frac{\partial^{2} u_{i}}{\partial t^{2}}-\sum_{j=1}^{n} \frac{\partial}{\partial x_{j}}\left(\sum_{\ell, m=1}^{n} a_{i j \ell m}(x) \frac{\partial u_{\ell}}{\partial x_{m}}\right)=f_{i}(x) & \text { in } \Omega \times(0, \infty), \\ \left.u_{i}\right|_{t=0}=u_{0, i}(x) & \text { in } \Omega, \\ \left.\frac{\partial u_{i}}{\partial t}\right|_{t=0}=u_{1, i}(x) & \text { in } \Omega, \\ \alpha(x) \sum_{j=1}^{n}\left(\sum_{\ell, m=1}^{n} a_{i j \ell m}(x) \frac{\partial u_{\ell}}{\partial x_{m}}\right) n_{j}(x)+(1-\alpha(x)) u_{i}(x)=0 & \text { on } \partial \Omega \times(0, \infty) .\end{cases}
$$

It should be noticed that our boundary condition

$$
\boldsymbol{B}_{\alpha} \boldsymbol{u}=\alpha(x)(\mathbf{a}(x) \cdot \boldsymbol{\nabla} \boldsymbol{u} \cdot \mathbf{n})+(1-\alpha(x)) \boldsymbol{u}
$$

is a smooth linear combination of displacement and traction boundary conditions. It is easy to see that $\boldsymbol{B}_{\alpha}$ is non-degenerate (or coercive) if and only if either $\alpha(x)>0$ on $\partial \Omega$ (the Robin case) or $\alpha(x) \equiv 0$ on $\partial \Omega$ (the Dirichlet case). MarsdenHughes [17] studied the non-degenerate case. More precisely, they assume that the boundary $\partial \Omega$ is the disjoint union of the two closed subsets $\Gamma_{0}=\{x \in \partial \Omega: \alpha(x)=$ $0\}$ and $\partial \Omega \backslash \Gamma_{0}=\{x \in \partial \Omega: \alpha(x)>0\}$.

However, our boundary condition $\boldsymbol{B}_{\alpha}$ is degenerate from an analytical point of view. This is due to the fact that the so-called Shapiro-Lopatinskii complementary condition is violated at the points $x \in \partial \Omega$ where $\alpha(x)=0$ (cf. [11]). For example, in the case where $n=3, \alpha(x)$ may be a function such that, in terms of local coordinates $\left(x_{1}, x_{2}\right)$ of $\partial \Omega$,

$$
\alpha(x)=e^{-1 / x_{1}^{2}} \sin ^{2} \frac{1}{x_{1}} e^{-1 / x_{2}^{2}} \sin ^{2} \frac{1}{x_{2}} .
$$


Therefore, the crucial point in our semigroup approach is how to generalize the classical variational approach to the degenerate case (see Subsection 2.1).

We give two simple but important examples of the initial-boundary value problem (1.1):

Example 1.1 If we take

$$
\mathbf{a}(x)=\left(a_{i j \ell m}(x)\right)=\left(\delta_{i \ell} \delta_{j m}\right),
$$

then the initial-boundary value problem (1.1) becomes as follows ([14], [15]):

$$
\begin{cases}\frac{\partial^{2} \boldsymbol{u}}{\partial t^{2}}-\Delta \boldsymbol{u}=\boldsymbol{f} & \text { in } \Omega \times(0, \infty) \\ \left.\boldsymbol{u}\right|_{t=0}=\boldsymbol{u}_{0} & \text { in } \Omega, \\ \left.\frac{\partial \boldsymbol{u}}{\partial t}\right|_{t=0}=\boldsymbol{u}_{1} & \text { in } \Omega \\ \alpha(x) \frac{\partial \boldsymbol{u}}{\partial \mathbf{n}}+(1-\alpha(x)) \boldsymbol{u}=\mathbf{0} & \text { on } \partial \Omega \times(0, \infty) .\end{cases}
$$

Example 1.2 If we take

$$
\mathbf{a}(x)=\left(a_{i j \ell m}(x)\right)=\left(\lambda \delta_{i j} \delta_{\ell m}+\mu \delta_{i \ell} \delta_{j m}\right),
$$

where $\lambda, \mu$ are Lamé moduli, then the initial-boundary value problem (1.1) becomes as follows $([16])$ :

$$
\begin{cases}\frac{\partial^{2} \boldsymbol{u}}{\partial t^{2}}-(\mu \Delta \boldsymbol{u}+(\lambda+\mu) \operatorname{grad}(\operatorname{div} \boldsymbol{u}))=\boldsymbol{f} & \text { in } \Omega \times(0, \infty), \\ \left.\boldsymbol{u}\right|_{t=0}=\boldsymbol{u}_{0} & \text { in } \Omega, \\ \left.\frac{\partial \boldsymbol{u}}{\partial t}\right|_{t=0}=\boldsymbol{u}_{1} & \text { in } \Omega, \\ \alpha(x)(\boldsymbol{\tau}(\boldsymbol{u}) \cdot \mathbf{n})+(1-\alpha(x)) \boldsymbol{u}=\mathbf{0} & \text { on } \partial \Omega \times(0, \infty) .\end{cases}
$$

Here

$$
\boldsymbol{\tau}(\boldsymbol{u})=\left(\tau_{i j}(x)\right)=\left(\lambda \sum_{k=1}^{n} e_{k k}(x) \delta_{i j}+2 \mu e_{i j}(x)\right)
$$

is the linearized stress tensor.

The purpose of this paper is to study the initial-boundary value problem (1.1) from the viewpoint of semigroup theory. To do this, we consider the following boundary value problem of linear elastostatics (see [17, Chapter 4, Section 4.3]): For given vector functions $\boldsymbol{g}(x)=\left(g_{i}(x)\right)$ and $\boldsymbol{\varphi}(x)=\left(\varphi_{i}(x)\right)$ defined in $\Omega$ and on $\partial \Omega$, respectively, find a vector function $\boldsymbol{v}(x)=\left(v_{i}(x)\right)$ in $\Omega$ such that

$$
\begin{cases}\operatorname{div}(\mathbf{a}(x) \cdot \boldsymbol{\nabla} \boldsymbol{v})=\boldsymbol{g} & \text { in } \Omega \\ \alpha(x)(\mathbf{a}(x) \cdot \boldsymbol{\nabla} \boldsymbol{v} \cdot \mathbf{n})+(1-\alpha(x)) \boldsymbol{v}=\boldsymbol{\varphi} & \text { on } \partial \Omega\end{cases}
$$

We study the boundary value problem (1.4) in the framework of $L^{2}$-spaces, by using the $L^{2}$ theory of pseudo-differential operators (see [20]).

If $s \in \mathbf{R}$, we let

$\boldsymbol{H}^{s}\left(\Omega, \mathbf{R}^{n}\right)=$ the Sobolev space of all $H^{s}$-vector functions $\boldsymbol{v}=\left(v_{i}\right)$ on $\Omega$,

$\boldsymbol{B}^{s}\left(\partial \Omega, \mathbf{R}^{n}\right)=$ the Besov space of all $B^{s}$-vector functions $\boldsymbol{\varphi}=\left(\varphi_{i}\right)$ on $\partial \Omega$. 
For the basic definitions and properties of Sobolev and Besov spaces, see AdamsFournier [1], Bergh-Löfström [2], Stein [18] and Triebel [22].

We introduce a subspace of $\boldsymbol{B}^{s}\left(\partial \Omega, \mathbf{R}^{n}\right)$ which is associated with the boundary condition

$$
\alpha(x)(\mathbf{a}(x) \cdot \boldsymbol{\nabla} \boldsymbol{v} \cdot \mathbf{n})+(1-\alpha(x)) \boldsymbol{v}=\boldsymbol{\varphi} \quad \text { on } \partial \Omega .
$$

If $s \in \mathbf{R}$, we let

$$
\begin{gathered}
\boldsymbol{B}_{(\alpha)}^{s}\left(\partial \Omega, \mathbf{R}^{n}\right)=\left\{\boldsymbol{\varphi}=\alpha(x) \boldsymbol{\varphi}_{1}+(1-\alpha(x)) \boldsymbol{\varphi}_{2}: \boldsymbol{\varphi}_{1} \in \boldsymbol{B}^{s}\left(\partial \Omega, \mathbf{R}^{n}\right),\right. \\
\left.\boldsymbol{\varphi}_{2} \in \boldsymbol{B}^{s+1}\left(\partial \Omega, \mathbf{R}^{n}\right)\right\}
\end{gathered}
$$

and define its norm

$$
\|\boldsymbol{\varphi}\|_{\alpha ; s}=\inf \left\{\left\|\boldsymbol{\varphi}_{1}\right\|_{\boldsymbol{B}^{s}\left(\partial \Omega, \mathbf{R}^{n}\right)}+\left\|\boldsymbol{\varphi}_{2}\right\|_{\boldsymbol{B}^{s+1}\left(\partial \Omega, \mathbf{R}^{n}\right)}: \boldsymbol{\varphi}=\alpha(x) \boldsymbol{\varphi}_{1}+(1-\alpha(x)) \boldsymbol{\varphi}_{2}\right\} .
$$

Then it is easy to verify (see $[19$, Lemma 4.7$])$ that the space $\boldsymbol{B}_{(\alpha)}^{s}\left(\partial \Omega, \mathbf{R}^{n}\right)$ is a Banach space with the norm $\|\cdot\|_{\alpha ; s}$. Furthermore, we remark that

$$
\begin{aligned}
& \boldsymbol{B}_{(\alpha)}^{s}\left(\partial \Omega, \mathbf{R}^{n}\right)=\boldsymbol{B}^{s+1}\left(\partial \Omega, \mathbf{R}^{n}\right) \quad \text { if } \alpha(x) \equiv 0 \text { on } \partial \Omega \text { (displacement), } \\
& \boldsymbol{B}_{(\alpha)}^{s}\left(\partial \Omega, \mathbf{R}^{n}\right)=\boldsymbol{B}^{s}\left(\partial \Omega, \mathbf{R}^{n}\right) \quad \text { if } \alpha(x) \equiv 1 \text { on } \partial \Omega \text { (traction), }
\end{aligned}
$$

and for general $\alpha(x)$ the continuous injections

$$
\boldsymbol{B}^{s+1}\left(\partial \Omega, \mathbf{R}^{n}\right) \subset \boldsymbol{B}_{(\alpha)}^{s}\left(\partial \Omega, \mathbf{R}^{n}\right) \subset \boldsymbol{B}^{s}\left(\partial \Omega, \mathbf{R}^{n}\right) .
$$

Now we let

$$
\begin{aligned}
& \boldsymbol{A} \boldsymbol{v}=\operatorname{div}(\mathbf{a}(x) \cdot \boldsymbol{\nabla} \boldsymbol{v}), \\
& \boldsymbol{B}_{\alpha} \boldsymbol{v}=\alpha(x)(\mathbf{a}(x) \cdot \boldsymbol{\nabla} \boldsymbol{v} \cdot \mathbf{n})+\left.(1-\alpha(x)) \boldsymbol{v}\right|_{\partial \Omega},
\end{aligned}
$$

and associate with problem (1.4) a linear operator

$$
\mathcal{A}=\left(\boldsymbol{A}, \boldsymbol{B}_{\alpha}\right): \boldsymbol{H}^{s}\left(\Omega, \mathbf{R}^{n}\right) \longrightarrow \boldsymbol{H}^{s-2}\left(\Omega, \mathbf{R}^{n}\right) \times \boldsymbol{B}_{(\alpha)}^{s-3 / 2}\left(\partial \Omega, \mathbf{R}^{n}\right) .
$$

Then it is easy to verify that the operator $\mathcal{A}$ is continuous for $s>3 / 2$.

Our starting point is the following existence and uniqueness theorem for the problem (1.4) due to Taira [20, Theorem 3.1] with $p:=2$ :

Theorem 1.1 Let $s>3 / 2$. We assume that the following two conditions ( $T)$ and $(A)$ are satisfied:

(T) The elasticity tensor $\mathbf{a}(x)=\left(a_{i j \ell m}(x)\right)$ enjoys the property of symmetry

$$
a_{i j \ell m}(x)=a_{\ell m i j}(x)=a_{j i \ell m}(x), \quad x \in \bar{\Omega},
$$

and is uniformly pointwise stable, that is, there is a constant $\eta>0$ such that

$$
\frac{1}{2} \boldsymbol{e} \cdot \mathbf{a}(x) \cdot \boldsymbol{e} \geq \eta\|\boldsymbol{e}\|^{2}, \quad x \in \bar{\Omega}
$$

for all symmetric two tensors $\boldsymbol{e}$.

(A) $0 \leq \alpha(x) \leq 1$ on $\partial \Omega$, but $\alpha(x) \not \equiv 1$ on $\partial \Omega$. 
Then the operator

$$
\mathcal{A}=\left(\boldsymbol{A}, \boldsymbol{B}_{\alpha}\right): \boldsymbol{H}^{s}\left(\Omega, \mathbf{R}^{n}\right) \longrightarrow \boldsymbol{H}^{s-2}\left(\Omega, \mathbf{R}^{n}\right) \times \boldsymbol{B}_{(\alpha)}^{s-3 / 2}\left(\partial \Omega, \mathbf{R}^{n}\right)
$$

is an algebraic and topological isomorphism. In particular, there exist constants $C_{1}>0$ and $C_{2}>0$ such that

$$
\begin{aligned}
C_{1}\|\boldsymbol{u}\|_{\boldsymbol{H}^{s}\left(\Omega, \mathbf{R}^{n}\right)} & \leq\|\boldsymbol{A} \boldsymbol{u}\|_{\boldsymbol{H}^{s-2}\left(\Omega, \mathbf{R}^{n}\right)}+\left\|\boldsymbol{B}_{\alpha} \boldsymbol{u}\right\|_{\alpha ; s-3 / 2} \\
& \leq C_{2}\|\boldsymbol{u}\|_{\boldsymbol{H}^{s}\left(\Omega, \mathbf{R}^{n}\right)} \quad \text { for all } \boldsymbol{u} \in \boldsymbol{H}^{s}\left(\Omega, \mathbf{R}^{n}\right) .
\end{aligned}
$$

Remark 1.1 Condition (A) implies that our boundary condition is not equal to the pure traction boundary condition. It is known (see [17, Chapter 7, Section 7.3]) that the pure traction problem may have non-unique solutions even for small loads and near a stress free state.

The rest of this paper is organized as follows. In Section 2 we introduce a linear operator $\mathfrak{A}$ in the Hilbert space $\boldsymbol{L}^{2}\left(\Omega, \mathbf{R}^{n}\right)=\boldsymbol{H}^{0}\left(\Omega, \mathbf{R}^{n}\right)$, and state that $\boldsymbol{-} \mathfrak{A}$ is a positive definite, self-adjoint operator (Theorem 2.1 ). Then we can define the square root $\mathfrak{C}=\sqrt{-\mathfrak{A}}$ of $-\mathfrak{A}$, and introduce an underlying Hilbert space $\boldsymbol{H}=D(\mathfrak{C})$. We state our fundamental existence and uniqueness theorem for the initial-boundary value problem (1.1) (Theorem 2.3). Theorem 2.1 is proved in Section 3 due to its length, and Theorem 2.3 is proved in a series of theorems (Theorem 5.3, Theorem 6.1, Theorem 7.1 and Theorem 8.1). In Section 4 we prove that the underlying Hilbert space $\boldsymbol{\mathcal { H }}$ is the right space for our variational approach (Theorem 4.1). Section 5 is devoted to a semigroup approach to the initial-boundary value problem (1.1) of linear elastodynamics. In particular, we prove the fundamental generation theorem for a strongly continuous group $e^{t \boldsymbol{A}}$ of unitary operators in the Hilbert space $\boldsymbol{X}=\boldsymbol{H} \times \boldsymbol{L}^{2}\left(\Omega, \mathbf{R}^{n}\right)$ (Theorem 5.1). By applying Theorem 5.1 to the initial-boundary value problem (1.1), we can obtain the representation formula of a (unique) solution of problem (1.1) (Theorem 5.3). This section is the heart of the subject. In Section 6 we prove the fundamental existence and uniqueness theorem for problem (1.1) (Theorem 6.1). In Section 7 we prove the fundamental energy inequality for problem (1.1) (Theorem 7.1). Section 8 is devoted to the regularity theorem for problem (1.1) (Theorem 8.1). In the last Section 9 we give two important open problems concerning the initial-boundary value problem (1.1) of linear elastodynamics.

The presentation on some results of this paper was given in "Conference on Evolution Equations and Semigroups" which was held between April 8 and 13, 2002 at Cortona, Italy.

\section{Statement of Main Results}

In this section we introduce a linear operator $\boldsymbol{A}$ in the Hilbert space $\boldsymbol{L}^{2}\left(\Omega, \mathbf{R}^{n}\right)$, and state that $-\boldsymbol{A}$ is a positive definite, self-adjoint operator (Theorem 2.1). Then we can define the square root $\mathfrak{C}=\sqrt{-\mathfrak{A}}$, and introduce an underlying Hilbert space $\boldsymbol{H}=D(\mathfrak{C})$ which is the right space for our variational approach. We state our fundamental existence and uniqueness theorem for the initial-boundary value problem (1.1) (Theorem 2.3). 


\subsection{Hilbert Space $\boldsymbol{H}$}

We introduce a linear operator $\boldsymbol{A}$ from the Hilbert space $\boldsymbol{L}^{2}\left(\Omega, \mathbf{R}^{n}\right)$ into itself as follows:

(1) The domain $D(\boldsymbol{A})$ is the space

$$
D(\mathfrak{A})=\left\{\boldsymbol{u} \in \boldsymbol{H}^{2}\left(\Omega, \mathbf{R}^{n}\right): \alpha(x)(\mathbf{a}(x) \cdot \boldsymbol{\nabla} \boldsymbol{u} \cdot \mathbf{n})+(1-\alpha(x)) \boldsymbol{u}=0 \text { on } \partial \Omega\right\} .
$$

(2) $\boldsymbol{A} \boldsymbol{u}=\operatorname{div}(\mathbf{a}(x) \cdot \boldsymbol{\nabla} \boldsymbol{u})$ for every $\boldsymbol{u} \in D(\mathfrak{A})$.

We remark that the domain $D(\boldsymbol{\mathfrak { A }})$ is dense in the space $\boldsymbol{L}^{2}\left(\Omega, \mathbf{R}^{n}\right)$.

Then we have the following fundamental result:

Theorem 2.1 The operator $-\mathfrak{A}$ is a positive definite, self-adjoint operator in the Hilbert space $\boldsymbol{L}^{2}\left(\Omega, \mathbf{R}^{n}\right)$.

Theorem 2.1 will be proved in the next Section 3 due to its length.

By virtue of Theorem 2.1, we have the following generation theorem for an analytic semigroup in the Hilbert space $\boldsymbol{L}^{2}\left(\Omega, \mathbf{R}^{n}\right)$ (cf. [19, Theorem 1.2]):

Theorem 2.2 The operator $\mathfrak{A}$ generates an analytic semigroup $e^{t \mathfrak{A}}$ in some sector containing the positive real axis.

Moreover, we can define the square root $\mathfrak{C}=\sqrt{-\mathfrak{A}}$ of the operator $-\mathfrak{A}$ by the formula (cf. [19, Theorem 1.10])

$$
\mathfrak{C} \boldsymbol{u}=-\frac{1}{\pi} \int_{0}^{\infty} s^{-1 / 2}(s I-\mathfrak{A})^{-1} \mathfrak{A} \boldsymbol{u} d s \quad \text { for all } \boldsymbol{u} \in D(\mathfrak{A})
$$

and introduce an underlying Hilbert space $\mathcal{H}$ as follows (cf. [21, Theorem 3.1]):

$$
\begin{aligned}
& \mathcal{H}=\text { the domain } D(\mathfrak{C}) \text { with the inner product } \\
& \qquad(\boldsymbol{u}, \boldsymbol{v})_{\mathcal{H}}=(\boldsymbol{C u}, \mathfrak{C} \boldsymbol{v})_{\boldsymbol{L}^{2}\left(\Omega, \mathbf{R}^{n}\right)}, \quad \boldsymbol{u}, \boldsymbol{v} \in D(\mathfrak{C}) .
\end{aligned}
$$

Remark 2.1 We have the following assertion for the non-degenerate case (see [6]):

$$
\mathcal{H}=D(\mathfrak{C})= \begin{cases}\boldsymbol{H}_{0}^{1}\left(\Omega, \mathbf{R}^{n}\right) & \text { if } \alpha(x) \equiv 0 \text { on } \partial \Omega(\text { the Dirichlet case }) \\ \boldsymbol{H}^{1}\left(\Omega, \mathbf{R}^{n}\right) & \text { if } \alpha(x)>0 \text { on } \partial \Omega(\text { the Robin case })\end{cases}
$$

where

$$
\boldsymbol{H}_{0}^{1}\left(\Omega, \mathbf{R}^{n}\right)=\left\{\boldsymbol{u} \in \boldsymbol{H}^{1}\left(\Omega, \mathbf{R}^{n}\right): \boldsymbol{u}=0 \text { on } \partial \Omega\right\}
$$

2.2 Existence and Uniqueness Theorem for Problem (1.1)

Now our fundamental existence and uniqueness theorem for the initial-boundary value problem (1.1) can be stated as follows: 
Theorem 2.3 Assume that conditions (T) and (A) are satisfied. Then, for any given data

$$
\boldsymbol{u}_{0} \in D(\boldsymbol{\mathfrak { A }}), \boldsymbol{u}_{1} \in \boldsymbol{H}, \boldsymbol{f}(t) \in C^{1}\left([0, \infty), \boldsymbol{L}^{2}\left(\Omega, \mathbf{R}^{n}\right)\right)
$$

the initial-boundary value problem (1.1) has a unique solution

$$
\boldsymbol{u}(t) \in C^{2}\left([0, \infty), \boldsymbol{L}^{2}\left(\Omega, \mathbf{R}^{n}\right)\right) \cap C^{1}([0, \infty), \boldsymbol{H}) \cap C([0, \infty), D(\mathfrak{A}))
$$

Moreover, the solution $\boldsymbol{u}(t)$ can be represented explicitly as the formula

$$
\boldsymbol{u}(t)=\cos (t \mathfrak{C}) \boldsymbol{u}_{0}+\mathfrak{C}^{-1} \sin (t \mathfrak{C}) \boldsymbol{u}_{1}+\int_{0}^{t} \mathfrak{C}^{-1} \sin ((t-s) \mathfrak{C}) \boldsymbol{f}(s) d s
$$

and we have the energy inequality

$$
\begin{aligned}
& \|\boldsymbol{u}(t)\|_{\boldsymbol{H}^{2}\left(\Omega, \mathbf{R}^{n}\right)}+\left\|\boldsymbol{u}^{\prime}(t)\right\|_{\mathcal{H}}+\left\|\boldsymbol{u}^{\prime \prime}(t)\right\|_{\boldsymbol{L}^{2}\left(\Omega, \mathbf{R}^{n}\right)} \\
\leq & C\left(\left\|\boldsymbol{u}_{0}\right\|_{\boldsymbol{H}^{2}\left(\Omega, \mathbf{R}^{n}\right)}+\left\|\boldsymbol{u}_{1}\right\|_{\mathcal{H}}+\|\boldsymbol{f}(0)\|_{\boldsymbol{L}^{2}\left(\Omega, \mathbf{R}^{n}\right)}+\int_{0}^{t}\left\|\boldsymbol{f}^{\prime}(s)\right\|_{\boldsymbol{L}^{2}\left(\Omega, \mathbf{R}^{n}\right)} d s\right),
\end{aligned}
$$

with a constant $C>0$ independent of $t$.

Theorem 2.3 will be proved in a series of theorems (Theorem 5.3, Theorem 6.1, Theorem 7.1 and Theorem 8.1).

We give two simple but important examples of Theorem 2.3 (cf. [14, Theorem I], [16, Theorem 3.9]):

Example 2.1 We let

$$
\mathbf{a}(x)=\left(a_{i j \ell m}(x)\right)=\left(\delta_{i \ell} \delta_{j m}\right) .
$$

Assume that condition (A) is satisfied. Then Theorem 2.3 applies to the mixed displacement-traction problem for the wave equation (1.2). Indeed, it is easy to verify that inequality (1.5) holds true with $\eta=1 / 2$.

Example 2.2 We let

$$
\mathbf{a}(x)=\left(a_{i j \ell m}(x)\right)=\left(\lambda \delta_{i j} \delta_{\ell m}+\mu \delta_{i \ell} \delta_{j m}\right),
$$

where $\lambda$ and $\mu$ are Lamé moduli. In addition to condition (A), assume that the following condition (L) is satisfied:

(L) $\mu>0$ and $2 \mu+n \lambda>0$.

Then Theorem 2.3 applies to the mixed displacement-traction problem for the elastodynamic wave equation (1.3). Indeed, it is easy to verify that inequality (1.5) holds true with

$$
\eta=\min \left\{\mu, \mu+\frac{n}{2} \lambda\right\}
$$

if condition (L) is satisfied (see [17, Chapter 4, Proposition 3.13]). 


\section{Proof of Theorem 2.1}

In this section we prove Theorem 2.1. By virtue of [25, Chapter VII, Section 3, Corollary]), we have only to prove the following three assertions:

(i) The operator $\boldsymbol{A}: D(\mathfrak{A}) \rightarrow \boldsymbol{L}^{2}\left(\Omega, \mathbf{R}^{n}\right)$ is bijective.

(ii) The operator $\boldsymbol{A}$ is symmetric in $\boldsymbol{L}^{2}\left(\Omega, \mathbf{R}^{n}\right)$.

(iii) The operator $-\boldsymbol{A}$ is non-negative in $\boldsymbol{L}^{2}\left(\Omega, \mathbf{R}^{n}\right)$.

Assertion (i) follows by applying Theorem 1.1 with $s:=2$ and $\boldsymbol{B}_{\alpha} \boldsymbol{u}=0$.

In order to prove assertion (ii), it suffices to show that

$$
(\boldsymbol{\mathfrak { A }} \boldsymbol{u}, \boldsymbol{v})_{\boldsymbol{L}^{2}\left(\Omega, \mathbf{R}^{n}\right)}=(\boldsymbol{u}, \mathfrak{A} \boldsymbol{v})_{\boldsymbol{L}^{2}\left(\Omega, \mathbf{R}^{n}\right)} \quad \text { for all } \boldsymbol{u}, \boldsymbol{v} \in D(\mathfrak{A}) .
$$

By applying the divergence theorem to our situation, we have the formulas

$$
\begin{aligned}
(\boldsymbol{u}, \mathfrak{A} \boldsymbol{v})_{\boldsymbol{L}^{2}\left(\Omega, \mathbf{R}^{n}\right)} & =\int_{\Omega} \boldsymbol{u} \cdot \operatorname{div}(\mathbf{a}(x) \cdot \boldsymbol{\nabla} \boldsymbol{v}) d x \\
& =\int_{\partial \Omega} \boldsymbol{u}[\mathbf{a}(x) \cdot \boldsymbol{\nabla} \boldsymbol{v} \cdot \mathbf{n}] d \sigma-\int_{\Omega} \boldsymbol{\nabla} \boldsymbol{u} \cdot \mathbf{a}(x) \cdot \boldsymbol{\nabla} \boldsymbol{v} d x
\end{aligned}
$$

and

$$
\begin{aligned}
(\boldsymbol{\mathfrak { A }}, \boldsymbol{v})_{\boldsymbol{L}^{2}\left(\Omega, \mathbf{R}^{n}\right)} & =\int_{\Omega} \boldsymbol{v} \cdot \operatorname{div}(\mathbf{a}(x) \cdot \boldsymbol{\nabla} \boldsymbol{u}) d x \\
& =\int_{\partial \Omega} \boldsymbol{v}[\mathbf{a}(x) \cdot \boldsymbol{\nabla} \boldsymbol{u} \cdot \mathbf{n}] d \sigma-\int_{\Omega} \boldsymbol{\nabla} \boldsymbol{v} \cdot \mathbf{a}(x) \cdot \boldsymbol{\nabla} \boldsymbol{u} d x,
\end{aligned}
$$

where $d \sigma$ is the area element on the boundary $\partial \Omega$.

However, by the symmetry of the tensor a $(x)$ it follows that

$$
\int_{\Omega} \boldsymbol{\nabla} \boldsymbol{u} \cdot \mathbf{a}(x) \cdot \boldsymbol{\nabla} \boldsymbol{v} d x=\int_{\Omega} \boldsymbol{\nabla} \boldsymbol{v} \cdot \mathbf{a}(x) \cdot \boldsymbol{\nabla} \boldsymbol{u} d x .
$$

Moreover, we have, for all $\boldsymbol{u}, \boldsymbol{v} \in D(\mathfrak{A})$,

$$
\begin{aligned}
\int_{\partial \Omega} \boldsymbol{u}[\mathbf{a}(x) \cdot \boldsymbol{\nabla} \boldsymbol{v} \cdot \mathbf{n}] d \sigma & =-\int_{\{\alpha(x) \neq 0\}} \boldsymbol{u} \cdot \frac{1-\alpha(x)}{\alpha(x)} \boldsymbol{v} d \sigma \\
& =-\int_{\{\alpha(x) \neq 0\}} \boldsymbol{v} \cdot \frac{1-\alpha(x)}{\alpha(x)} \boldsymbol{u} d \sigma \\
& =\int_{\partial \Omega} \boldsymbol{v}[\mathbf{a}(x) \cdot \boldsymbol{\nabla} \boldsymbol{u} \cdot \mathbf{n}] d \sigma .
\end{aligned}
$$

Here it should be emphasized that the boundary integral

$$
\int_{\{\alpha(x) \neq 0\}} \boldsymbol{u} \cdot \frac{1-\alpha(x)}{\alpha(x)} \boldsymbol{v} d \sigma
$$

is finite. Indeed, it follows from formulas (3.5) and (3.2) that

$$
\begin{aligned}
-\int_{\{\alpha(x) \neq 0\}} \boldsymbol{u} \cdot \frac{1-\alpha(x)}{\alpha(x)} \boldsymbol{v} d \sigma & =\int_{\partial \Omega} \boldsymbol{u}[\mathbf{a}(x) \cdot \boldsymbol{\nabla} \boldsymbol{v} \cdot \mathbf{n}] d \sigma \\
& =(\boldsymbol{u}, \mathfrak{A} \boldsymbol{v})_{\boldsymbol{L}^{2}\left(\Omega, \mathbf{R}^{n}\right)}+\int_{\Omega} \boldsymbol{\nabla} \boldsymbol{u} \cdot \mathbf{a}(x) \cdot \boldsymbol{\nabla} \boldsymbol{v} d x
\end{aligned}
$$


where $\boldsymbol{u}, \boldsymbol{A} \boldsymbol{v} \in \boldsymbol{L}^{2}\left(\Omega, \mathbf{R}^{n}\right)$ and $\boldsymbol{\nabla} \boldsymbol{u}, \boldsymbol{\nabla} \boldsymbol{v} \in H^{1}\left(\Omega, \mathbf{R}^{n^{2}}\right)$.

Therefore, by using formulas (3.4) and (3.5) we obtain from formulas (3.2) and (3.3) that

$$
\begin{aligned}
(\boldsymbol{u}, \mathfrak{A} \boldsymbol{v})_{\boldsymbol{L}^{2}\left(\Omega, \mathbf{R}^{n}\right)} & =\int_{\partial \Omega} \boldsymbol{u}[\mathbf{a}(x) \cdot \boldsymbol{\nabla} \boldsymbol{v} \cdot \mathbf{n}] d \sigma-\int_{\Omega} \boldsymbol{\nabla} \boldsymbol{u} \cdot \mathbf{a}(x) \cdot \boldsymbol{\nabla} \boldsymbol{v} d x \\
& =\int_{\partial \Omega} \boldsymbol{v}[\mathbf{a}(x) \cdot \boldsymbol{\nabla} \boldsymbol{u} \cdot \mathbf{n}] d \sigma-\int_{\Omega} \boldsymbol{\nabla} \boldsymbol{v} \cdot \mathbf{a}(x) \cdot \boldsymbol{\nabla} \boldsymbol{u} d x \\
& =\int_{\Omega} \boldsymbol{v} \cdot \operatorname{div}(\mathbf{a}(x) \cdot \boldsymbol{\nabla} \boldsymbol{u}) d x \\
& =(\mathfrak{A} \boldsymbol{u}, \boldsymbol{v})_{\boldsymbol{L}^{2}\left(\Omega, \mathbf{R}^{n}\right)} \quad \text { for all } \boldsymbol{u}, \boldsymbol{v} \in D(\mathfrak{A}) .
\end{aligned}
$$

This proves the desired formula (3.1).

Finally, it remains to prove assertion (iii). To do this, we have, by formulas (3.3) and (3.5),

$$
\begin{aligned}
-(\boldsymbol{A} \boldsymbol{u}, \boldsymbol{u})_{\boldsymbol{L}^{2}\left(\Omega, \mathbf{R}^{n}\right)} & =-\int_{\partial \Omega} \boldsymbol{u}[\mathbf{a}(x) \cdot \boldsymbol{\nabla} \boldsymbol{u} \cdot \mathbf{n}] d \sigma+\int_{\Omega} \boldsymbol{\nabla} \boldsymbol{u} \cdot \mathbf{a}(x) \cdot \boldsymbol{\nabla} \boldsymbol{u} d x \\
& =\int_{\{\alpha(x) \neq 0\}} \frac{1-\alpha(x)}{\alpha(x)}\|\boldsymbol{u}\|^{2} d \sigma+\int_{\Omega} \boldsymbol{e}(\boldsymbol{u}) \cdot \mathbf{a}(x) \cdot \boldsymbol{e}(\boldsymbol{u}) d x,
\end{aligned}
$$

where

$$
\boldsymbol{e}(\boldsymbol{u})=\left(e_{i j}(x)\right)=\left(\frac{1}{2}\left(\frac{\partial u_{i}}{\partial x_{j}}+\frac{\partial u_{j}}{\partial x_{i}}\right)\right)
$$

is the linearized strain tensor. Therefore, by using inequality (1.5) we find from formula (3.6) that

$$
\begin{aligned}
(-\boldsymbol{A} \boldsymbol{u}, \boldsymbol{u})_{\boldsymbol{L}^{2}\left(\Omega, \mathbf{R}^{n}\right)} & =\int_{\{\alpha(x) \neq 0\}} \frac{1-\alpha(x)}{\alpha(x)}\|\boldsymbol{u}\|^{2} d \sigma+\int_{\Omega} \boldsymbol{e}(\boldsymbol{u}) \cdot \mathbf{a}(x) \cdot \boldsymbol{e}(\boldsymbol{u}) d x \\
& \geq 2 \eta \int_{\Omega}\|\boldsymbol{e}(\boldsymbol{u})\|^{2} d x \\
& \geq 0 \quad \text { for all } \boldsymbol{u} \in D(\mathfrak{A}) .
\end{aligned}
$$

This proves the non-negativity of $-\mathfrak{A}$.

The proof of Theorem 2.1 is complete.

\section{Characterization of the Hilbert Space $\mathcal{H}$}

In this section we prove that the underlying Hilbert space $\mathcal{H}$ is the right space for our variational approach (Theorem 4.1). To do this, we prove the following characterization of $\mathcal{H}$ in terms of the domain $D(\mathfrak{A})$ (cf. [24, Definition 1], [7, Lemma 3.3]):

Proposition 4.1 The Hilbert space $\mathcal{H}$ coincides with the completion of the domain $D(\mathfrak{A})$ with respect to the inner product

$$
\begin{aligned}
(\boldsymbol{u}, \boldsymbol{v})_{\mathcal{H}} & =(-\boldsymbol{A} \boldsymbol{u}, \boldsymbol{v})_{\boldsymbol{L}^{2}\left(\Omega, \mathbf{R}^{n}\right)} \\
& =\int_{\Omega} \boldsymbol{\nabla} \boldsymbol{u} \cdot \mathbf{a}(x) \cdot \boldsymbol{\nabla} \boldsymbol{v} d x+\int_{\{\alpha(x) \neq 0\}} \frac{1-\alpha(x)}{\alpha(x)} \boldsymbol{u} \cdot \boldsymbol{v} d \sigma, \quad \boldsymbol{u}, \boldsymbol{v} \in D(\mathfrak{A}) .
\end{aligned}
$$


Proof (1) Formula (4.1) follows by combining formulas (3.3) and (3.5) if we take $\boldsymbol{u}=\boldsymbol{v}$.

(2) We prove that the domain $D(\mathfrak{A})$ is dense in $D(\sqrt{-\mathfrak{A}})=D(\mathfrak{C})$. To do this, we remark (see [19, Section 1.2]) that the operators

$$
\mathfrak{C}^{-1}: \boldsymbol{L}^{2}\left(\Omega, \mathbf{R}^{n}\right) \longrightarrow D(\mathfrak{C})=\mathcal{H}
$$

and

$$
\mathfrak{C}^{-1}: D(\mathfrak{C}) \longrightarrow D\left(\mathfrak{C}^{2}\right)=D(\mathfrak{A})
$$

are algebraic and topological isomorphisms, and further that

$$
D\left(\mathfrak{C}^{3}\right) \subset D(\mathfrak{A})
$$

Therefore, we obtain that the domain $D(\mathfrak{A})$ is dense in the domain $D(\mathfrak{C})$, since $D(\mathfrak{A})$ is dense in the space $\boldsymbol{L}^{2}\left(\Omega, \mathbf{R}^{n}\right)$.

The situation can be visualized in the following diagram:

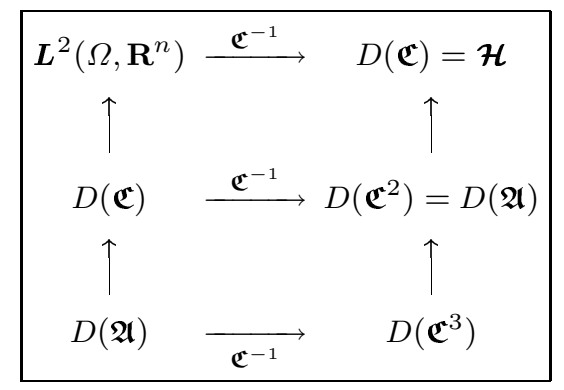

The proof of Proposition 4.1 is complete.

The next theorem shows that the Hilbert space $\mathcal{H}=D(\mathfrak{C})$ is the right space for the variational approach:

Theorem 4.1 We have the inclusions

$$
D(\mathfrak{A}) \subset \boldsymbol{H} \subset \boldsymbol{H}^{1}\left(\Omega, \mathbf{R}^{n}\right),
$$

with continuous injections.

Proof It suffices to prove that the inclusion $\boldsymbol{H} \subset \boldsymbol{H}^{1}\left(\Omega, \mathbf{R}^{n}\right)$ is continuous. The proof is divided into three steps.

Step 1: First, it follows from an application of the Rayleigh principle that we have, for all $u \in D(\boldsymbol{\mathfrak { A }})$,

$$
\begin{aligned}
\|\boldsymbol{u}\|_{\mathcal{H}}^{2} & =\|\boldsymbol{C} \boldsymbol{u}\|_{\boldsymbol{L}^{2}\left(\Omega, \mathbf{R}^{n}\right)}^{2}=\left(\mathfrak{C}^{2} \boldsymbol{u}, \boldsymbol{u}\right)_{\boldsymbol{L}^{2}\left(\Omega, \mathbf{R}^{n}\right)}=(-\boldsymbol{A} \boldsymbol{u}, \boldsymbol{u})_{\boldsymbol{L}^{2}\left(\Omega, \mathbf{R}^{n}\right)} \\
& \geq \lambda_{1}\|\boldsymbol{u}\|_{\boldsymbol{L}^{2}\left(\Omega, \mathbf{R}^{n}\right)}^{2}
\end{aligned}
$$

where $\lambda_{1}>0$ is the first eigenvalue of the operator $-\boldsymbol{A}$ (see Theorem 2.1).

However, it follows from inequality (3.7) that

$$
(-\boldsymbol{A} \boldsymbol{u}, \boldsymbol{u})_{\boldsymbol{L}^{2}\left(\Omega, \mathbf{R}^{n}\right)}=\int_{\{\alpha(x) \neq 0\}} \frac{1-\alpha(x)}{\alpha(x)}\|\boldsymbol{u}\|^{2} d \sigma+\int_{\Omega} \boldsymbol{e}(\boldsymbol{u}) \cdot \mathbf{a}(x) \cdot \boldsymbol{e}(\boldsymbol{u}) d x
$$




$$
\geq 2 \eta \int_{\Omega}\|\boldsymbol{e}(\boldsymbol{u})\|^{2} d x \quad \text { for all } \boldsymbol{u} \in D(\mathfrak{A})
$$

Step 2: The next inequalities are special cases of Gårding's inequality for the elliptic operator $\boldsymbol{u} \mapsto \boldsymbol{e}(\boldsymbol{u})$ (cf. [5, Chapitre 3, Théorèmes 3.1 et 3.3]):

Theorem 4.2 (Korn's inequalities) (i) For every non-empty open subset $\gamma \subset \partial \Omega$, there exists a constant $c(\gamma)>0$ such that

$$
\int_{\Omega}\|\boldsymbol{e}(\boldsymbol{u})\|^{2} d x \geq c(\gamma)\left(\int_{\Omega}\|\boldsymbol{u}\|^{2} d x+\int_{\Omega}\|\boldsymbol{\nabla} \boldsymbol{u}\|^{2} d x\right)
$$

for all $\boldsymbol{u} \in \boldsymbol{H}^{1}\left(\Omega, \mathbf{R}^{n}\right)$ satisfying the condition $\boldsymbol{u}=\mathbf{0}$ on $\gamma$.

(ii) There exists a constant $c>0$ such that

$$
\int_{\Omega}\|\boldsymbol{e}(\boldsymbol{u})\|^{2} d x+\int_{\Omega}\|\boldsymbol{u}\|^{2} d x \geq c\left(\int_{\Omega}\|\boldsymbol{u}\|^{2} d x+\int_{\Omega}\|\boldsymbol{\nabla} \boldsymbol{u}\|^{2} d x\right)
$$

for all $\boldsymbol{u} \in \boldsymbol{H}^{1}\left(\Omega, \mathbf{R}^{n}\right)$.

Step 3: By inequality (4.4) and Korn's inequality (4.6), it follows that

$$
\begin{aligned}
(-\mathfrak{A} \boldsymbol{u}, \boldsymbol{u})_{\boldsymbol{L}^{2}\left(\Omega, \mathbf{R}^{n}\right)} & \geq 2 \eta \int_{\Omega}\|\boldsymbol{e}(\boldsymbol{u})\|^{2} d x \\
& \geq 2 \eta c\|\boldsymbol{u}\|_{\boldsymbol{H}^{1}\left(\Omega, \mathbf{R}^{n}\right)}^{2}-2 \eta\|\boldsymbol{u}\|_{\boldsymbol{L}^{2}\left(\Omega, \mathbf{R}^{n}\right)}^{2} .
\end{aligned}
$$

Therefore, by combining inequalities (4.3) and (4.7) we obtain that

$$
\left(\frac{\lambda_{1}}{2 \eta}+1\right)(-\boldsymbol{A} \boldsymbol{u}, \boldsymbol{u})_{\boldsymbol{L}^{2}\left(\Omega, \mathbf{R}^{n}\right)} \geq\left(\lambda_{1} c\right)\|\boldsymbol{u}\|_{\boldsymbol{H}^{1}\left(\Omega, \mathbf{R}^{n}\right)}^{2} \quad \text { for all } \boldsymbol{u} \in D(\mathfrak{A}),
$$

so that

$$
\begin{aligned}
\|\boldsymbol{u}\|_{\mathcal{H}}^{2} & =(-\mathfrak{A} \boldsymbol{u}, \boldsymbol{u})_{\boldsymbol{L}^{2}\left(\Omega, \mathbf{R}^{n}\right)} \\
& \geq\left(\frac{\lambda_{1} c}{\lambda_{1} /(2 \eta)+1}\right)\|\boldsymbol{u}\|_{\boldsymbol{H}^{1}\left(\Omega, \mathbf{R}^{n}\right)}^{2} \quad \text { for all } \boldsymbol{u} \in D(\boldsymbol{A}) .
\end{aligned}
$$

This proves that the injection $\boldsymbol{H} \rightarrow \boldsymbol{H}^{1}\left(\Omega, \mathbf{R}^{n}\right)$ is continuous, since the domain $D(\mathfrak{A})$ is dense in the space $\mathcal{H}$.

The proof of Theorem 4.1 is complete.

\section{Semigroup Approach to Problem (1.1)}

This section is devoted to a semigroup approach to the initial-boundary value problem (1.1) of linear elastodynamics (Theorem 5.1). In particular, we prove the fundamental generation theorem for a strongly continuous group $e^{t \boldsymbol{A}}$ of unitary operators in the Hilbert space $\boldsymbol{X}=\boldsymbol{H} \times \boldsymbol{L}^{2}\left(\Omega, \mathbf{R}^{n}\right)$ (Theorem 5.1). By applying Theorem 5.1 to the initial-boundary value problem (1.1), we can obtain the representation formula of a (unique) solution of problem (1.1) (Theorem 5.3). This section is the heart of the subject. 
By using the operator $\mathfrak{A}$, we can formulate problem (1.1) in the abstract form

$$
\left\{\begin{array}{l}
\boldsymbol{u}^{\prime \prime}(t)=\boldsymbol{\mathfrak { u }} \boldsymbol{u}+\boldsymbol{f}(t) \quad t>0 \\
\boldsymbol{u}(0)=\boldsymbol{u}_{0} \\
\boldsymbol{u}^{\prime}(0)=\boldsymbol{u}_{1}
\end{array}\right.
$$

Furthermore, if we let

$$
\boldsymbol{X}=\boldsymbol{H} \times \boldsymbol{L}^{2}\left(\Omega, \mathbf{R}^{n}\right)
$$

and

$$
\boldsymbol{U}(t)=\left(\begin{array}{c}
\boldsymbol{u}(t) \\
\boldsymbol{u}^{\prime}(t)
\end{array}\right), \quad \boldsymbol{U}_{0}=\left(\begin{array}{c}
\boldsymbol{u}_{0} \\
\boldsymbol{u}_{1}
\end{array}\right), \quad \boldsymbol{F}(t)=\left(\begin{array}{c}
\mathbf{0} \\
\boldsymbol{f}(t)
\end{array}\right),
$$

and introduce a linear operator

$$
\boldsymbol{A}=\left(\begin{array}{cc}
\mathbf{0} & \boldsymbol{I} \\
\mathfrak{x} & 0
\end{array}\right): \mathcal{X} \longrightarrow \mathcal{X}
$$

with domain

$$
D(\boldsymbol{A})=D(\boldsymbol{\mathfrak { A }}) \times \boldsymbol{\mathcal { H }}
$$

then we can rewrite the initial value problem (5.1) as follows:

$$
\left\{\begin{array}{l}
\boldsymbol{U}^{\prime}(t)=\boldsymbol{A U}(t)+\boldsymbol{F}(t), \quad t>0 \\
\boldsymbol{U}(0)=\boldsymbol{U}_{0}
\end{array}\right.
$$

The fundamental generation theorem for groups can be stated as follows (cf. [24, Theorem 1], [8, Chapter II, Theorem 7.4]):

Theorem 5.1 The operator $\boldsymbol{A}$ generates a strongly continuous group $e^{t \boldsymbol{A}}$ of unitary operators in the Hilbert space $\boldsymbol{X}=\boldsymbol{H} \times \boldsymbol{L}^{2}\left(\Omega, \mathbf{R}^{n}\right)$. More precisely, the group $e^{\text {tA }}$ can be represented explicitly in the form

$$
e^{t \boldsymbol{A}}=\cos (t \mathfrak{C})\left(\begin{array}{ll}
\boldsymbol{I} & \mathbf{0} \\
\mathbf{0} & \boldsymbol{I}
\end{array}\right)+\mathfrak{C}^{-1} \sin (t \mathfrak{C})\left(\begin{array}{cc}
\mathbf{0} & \boldsymbol{I} \\
\mathfrak{A} & 0
\end{array}\right) .
$$

The proof of Theorem 5.1 is based on the following generation theorem for groups of class $\left(C_{0}\right)$ in a Banach space (see [10, Chapter XXII], [25, Chapter IX, Section 9, Corollary 1]):

Theorem 5.2 Let $A$ be a linear operator with dense domain $D(A)$ and range $R(A)$ both in a Banach space $X$. Assume that the resolvent $(n I-A)^{-1}$ exists and satisfies the condition

$$
\left\|(n I-A)^{-1}\right\| \leq \frac{1}{|n|} \quad \text { for every large non-zero integer }|n| .
$$

Then $A$ is the infinitesimal generator of a group $\left\{S_{t}\right\}$ of class $\left(C_{0}\right)$ of contraction operators in $X$. 
Proof The proof of Theorem 5.1 is divided into seven steps.

Step 1: First, we show that the operator $\boldsymbol{A}$ is densely defined, that is, the domain $D(\boldsymbol{A})$ is dense in the space $\boldsymbol{H}$.

By Proposition 4.1, it follows that the domain $D(\mathfrak{A})$ is dense in $\mathcal{H}$. Since the domain $D(\boldsymbol{A})$ is dense in the space $\boldsymbol{L}^{2}\left(\Omega, \mathbf{R}^{n}\right)$, we find that the domain $D(\boldsymbol{A})=$ $D(\mathfrak{A}) \times \mathcal{H}$ is dense in the product space $\boldsymbol{X}=\boldsymbol{H} \times \boldsymbol{L}^{2}\left(\Omega, \mathbf{R}^{n}\right)$.

Step 2: We show that $\boldsymbol{A}$ is a closed operator.

To do this, let $\left\{\boldsymbol{U}_{j}\right\}=\left\{\left(\boldsymbol{u}_{j}, \boldsymbol{v}_{j}\right)\right\}$ be a sequence in the domain $D(\boldsymbol{A})=D(\boldsymbol{A}) \times \boldsymbol{H}$ and let $\boldsymbol{U}=(\boldsymbol{u}, \boldsymbol{v})$ and $\boldsymbol{F}=(\boldsymbol{f}, \boldsymbol{g})$ be functions of $\boldsymbol{\mathcal { H }}=\boldsymbol{\mathcal { H }} \times \boldsymbol{L}^{2}\left(\Omega, \mathbf{R}^{n}\right)$ such that

$$
\begin{aligned}
& \boldsymbol{U}_{j}=\left(\begin{array}{l}
\boldsymbol{u}_{j} \\
\boldsymbol{v}_{j}
\end{array}\right) \longrightarrow \boldsymbol{U}=\left(\begin{array}{l}
\boldsymbol{u} \\
\boldsymbol{v}
\end{array}\right) \quad \text { in } \boldsymbol{X} \\
& \boldsymbol{A} \boldsymbol{U}_{j}=\left(\begin{array}{ll}
\mathbf{0} & \boldsymbol{I} \\
\mathfrak{A} & 0
\end{array}\right)\left(\begin{array}{l}
\boldsymbol{u}_{j} \\
\boldsymbol{v}_{j}
\end{array}\right) \longrightarrow \boldsymbol{F}=\left(\begin{array}{l}
\boldsymbol{f} \\
\boldsymbol{g}
\end{array}\right) \text { in } \mathcal{H} .
\end{aligned}
$$

Then we have the assertions

$$
\begin{cases}\boldsymbol{u}_{j} \longrightarrow \boldsymbol{u} & \text { in } \boldsymbol{H} \\ \boldsymbol{v}_{j} \longrightarrow \boldsymbol{v} & \text { in } \boldsymbol{L}^{2}\left(\Omega, \mathbf{R}^{n}\right)\end{cases}
$$

and

$$
\begin{cases}\boldsymbol{v}_{j} \longrightarrow \boldsymbol{f} & \text { in } \boldsymbol{H} \\ \mathfrak{A} \boldsymbol{u}_{j} \longrightarrow \boldsymbol{g} & \text { in } \boldsymbol{L}^{2}\left(\Omega, \mathbf{R}^{n}\right)\end{cases}
$$

However, it follows from an application of inequality (1.6) with $s:=2$ and $\boldsymbol{B}_{\alpha} \boldsymbol{u}=0$ that the operator

$$
\boldsymbol{A}: D(\mathfrak{A}) \longrightarrow \boldsymbol{L}^{2}\left(\Omega, \mathbf{R}^{n}\right)
$$

is isomorphic. Here the domain $D(\mathfrak{A})$ is a Banach space endowed with the graph norm

$$
\|\boldsymbol{u}\|_{D(\mathfrak{A})}=\|\boldsymbol{u}\|_{\boldsymbol{L}^{2}\left(\Omega, \mathbf{R}^{n}\right)}+\|\boldsymbol{A} \boldsymbol{u}\|_{\boldsymbol{L}^{2}\left(\Omega, \mathbf{R}^{n}\right)}, \quad \boldsymbol{u} \in D(\mathfrak{A}) .
$$

It should be noticed that the graph norm $\|\cdot\|_{D(\mathfrak{A})}$ is equivalent to the norm $\|\cdot\|_{\boldsymbol{H}^{2}\left(\Omega, \mathbf{R}^{n}\right)}$. In fact, we have the inequalities

$$
\begin{aligned}
& C_{1}\|\boldsymbol{u}\|_{\boldsymbol{H}^{2}\left(\Omega, \mathbf{R}^{n}\right)} \leq\|\boldsymbol{\mathcal { A }} \boldsymbol{u}\|_{\boldsymbol{L}^{2}\left(\Omega, \mathbf{R}^{n}\right)} \leq\|\boldsymbol{u}\|_{D(\boldsymbol{A})}, \\
& \|\boldsymbol{u}\|_{D(\boldsymbol{A})} \leq\|\boldsymbol{u}\|_{\boldsymbol{L}^{2}\left(\Omega, \mathbf{R}^{n}\right)}+C_{2}\|\boldsymbol{u}\|_{\boldsymbol{H}^{2}\left(\Omega, \mathbf{R}^{n}\right)} \leq\left(C_{2}+1\right)\|\boldsymbol{u}\|_{\boldsymbol{H}^{2}\left(\Omega, \mathbf{R}^{n}\right)} .
\end{aligned}
$$

Since $\left\{\boldsymbol{u}_{j}\right\}$ is a Cauchy the sequence in $\boldsymbol{H}^{2}\left(\Omega, \mathbf{R}^{n}\right)$, we can find a function $\boldsymbol{w} \in \boldsymbol{H}^{2}\left(\Omega, \mathbf{R}^{n}\right)$ such that

$$
\boldsymbol{u}_{j} \longrightarrow \boldsymbol{w} \quad \text { in } \boldsymbol{H}^{2}\left(\Omega, \mathbf{R}^{n}\right)
$$

Then it follows that

$$
\boldsymbol{A} \boldsymbol{w}=\lim _{j \rightarrow \infty} \boldsymbol{\mathfrak { A }} \boldsymbol{u}_{j}=\boldsymbol{g} \quad \text { in } \boldsymbol{L}^{2}\left(\Omega, \mathbf{R}^{n}\right)
$$

and further that

$$
\boldsymbol{B}_{\alpha} \boldsymbol{w}=\lim _{j \rightarrow \infty} \boldsymbol{B}_{\alpha} \boldsymbol{u}_{j}=0 \quad \text { in } \boldsymbol{B}^{1 / 2}\left(\partial \Omega, \mathbf{R}^{n}\right)
$$


This proves that

$$
\boldsymbol{u}=\boldsymbol{w} \in D(\boldsymbol{A})=\left\{\boldsymbol{v} \in \boldsymbol{H}^{2}\left(\Omega, \mathbf{R}^{n}\right): \boldsymbol{B}_{\alpha} \boldsymbol{v}=0 \text { on } \partial \Omega\right\},
$$

since $\boldsymbol{u}_{j} \rightarrow \boldsymbol{u}$ in $\mathcal{H}$.

Therefore, we obtain from assertions (5.5), (5.6) and (5.7) that

$$
\left\{\begin{array}{l}
\boldsymbol{u} \in D(\boldsymbol{\mathfrak { A }}), \\
\mathfrak{A} \boldsymbol{u}=\boldsymbol{g} \in \boldsymbol{L}^{2}\left(\Omega, \mathbf{R}^{n}\right) .
\end{array}\right.
$$

Moreover, since the injection $\boldsymbol{H} \subset \boldsymbol{L}^{2}\left(\Omega, \mathbf{R}^{n}\right)$ is continuous, it follows that

$$
\boldsymbol{v}_{j} \longrightarrow \boldsymbol{f} \quad \text { in } \boldsymbol{L}^{2}\left(\Omega, \mathbf{R}^{n}\right)
$$

so that

$$
\boldsymbol{v}=\boldsymbol{f} \in \mathcal{H} .
$$

Summing up, we have proved that

$$
\begin{aligned}
& \boldsymbol{U}=\left(\begin{array}{l}
\boldsymbol{u} \\
\boldsymbol{v}
\end{array}\right) \in D(\boldsymbol{A})=D(\mathfrak{A}) \times \mathcal{H} \\
& \boldsymbol{A} \boldsymbol{U}=\left(\begin{array}{c}
\boldsymbol{v} \\
\boldsymbol{A} \boldsymbol{u}
\end{array}\right)=\left(\begin{array}{l}
\boldsymbol{f} \\
\boldsymbol{g}
\end{array}\right)=\boldsymbol{F} .
\end{aligned}
$$

This proves the closedness of $\boldsymbol{A}$.

Step 3: We show that the operator $\lambda \boldsymbol{I}-\boldsymbol{A}$ is surjective for every $\lambda \neq 0$.

Let $\boldsymbol{F}=(\boldsymbol{f}, \boldsymbol{g})$ be an arbitrary function of $\boldsymbol{X}=\boldsymbol{H} \times \boldsymbol{L}^{2}\left(\Omega, \mathbf{R}^{n}\right)$. Then we have, for some function $\boldsymbol{U}=(\boldsymbol{u}, \boldsymbol{v})$ of $\boldsymbol{\mathcal { X }}$,

$$
\begin{aligned}
(\lambda \boldsymbol{I}-\boldsymbol{A}) \boldsymbol{U}=\boldsymbol{F} & \Longleftrightarrow\left\{\begin{array}{l}
\boldsymbol{u} \in D(\mathfrak{A}), \boldsymbol{v} \in \mathcal{H}, \\
\lambda \boldsymbol{u}-\boldsymbol{v}=\boldsymbol{f},-\boldsymbol{A} \boldsymbol{u}+\lambda \boldsymbol{v}=\boldsymbol{g}
\end{array}\right. \\
& \Longleftrightarrow\left\{\begin{array}{l}
\boldsymbol{u} \in D(\mathfrak{A}), \boldsymbol{v}=\lambda \boldsymbol{u}-\boldsymbol{f}, \\
\left(\lambda^{2}-\mathfrak{A}\right) \boldsymbol{u}=\lambda \boldsymbol{f}+\boldsymbol{g} .
\end{array}\right.
\end{aligned}
$$

However, since $\lambda^{2}>0$, it follows from an application of Theorem 2.1 that there exists a unique function $\boldsymbol{u} \in D(\mathfrak{A})$ such that

$$
\left(\lambda^{2}-\boldsymbol{A}\right) \boldsymbol{u}=\lambda \boldsymbol{f}+\boldsymbol{g} \in \boldsymbol{L}^{2}\left(\Omega, \mathbf{R}^{n}\right) .
$$

Hence, by letting

$$
\boldsymbol{v}=\lambda \boldsymbol{u}-\boldsymbol{f} \in \mathcal{H}
$$

we obtain that

$$
\boldsymbol{U}=\left(\begin{array}{l}
\boldsymbol{u} \\
\boldsymbol{v}
\end{array}\right) \in D(\boldsymbol{A})=D(\mathfrak{A}) \times \boldsymbol{\mathcal { H }}
$$

and that

$$
(\lambda \boldsymbol{I}-\boldsymbol{A}) \boldsymbol{U}=\boldsymbol{F} .
$$

This proves the $\lambda \boldsymbol{I}-\boldsymbol{A}$ is surjective for every $\lambda \neq 0$.

Step 4: We show that the operator $\boldsymbol{A}$ is dissipative. More precisely, we have, for all $\boldsymbol{U}, \boldsymbol{V} \in D(\boldsymbol{A})=D(\mathfrak{A}) \times \mathcal{H}$,

$$
(\boldsymbol{A} \boldsymbol{U}, \boldsymbol{V})_{\mathcal{X}}=-(\boldsymbol{U}, \boldsymbol{A} \boldsymbol{V})_{\mathcal{X}}
$$


In particular, we have, for all $\boldsymbol{U} \in D(\boldsymbol{A})$,

$$
(\boldsymbol{A U}, \boldsymbol{U})_{\mathcal{X}}=0
$$

This proves that $\boldsymbol{A}$ is dissipative.

If we let

$$
\boldsymbol{U}=\left(\begin{array}{l}
\boldsymbol{u}_{1} \\
\boldsymbol{v}_{1}
\end{array}\right), \quad \boldsymbol{V}=\left(\begin{array}{l}
\boldsymbol{u}_{2} \\
\boldsymbol{v}_{2}
\end{array}\right) \in D(\boldsymbol{A})=D(\boldsymbol{\mathfrak { A }}) \times \boldsymbol{H},
$$

then we have the formula

$$
\begin{aligned}
(\boldsymbol{A} \boldsymbol{U}, \boldsymbol{V})_{\mathcal{X}}= & \left(\left(\begin{array}{c}
\boldsymbol{v}_{1} \\
\boldsymbol{2} \boldsymbol{u}_{1}
\end{array}\right),\left(\begin{array}{c}
\boldsymbol{u}_{2} \\
\boldsymbol{v}_{2}
\end{array}\right)\right)_{\mathcal{X}} \\
= & \int_{\Omega} \boldsymbol{\nabla} \boldsymbol{v}_{1} \cdot \mathbf{a}(x) \cdot \boldsymbol{\nabla} \boldsymbol{u}_{2} d x+\int_{\{\alpha(x) \neq 0\}} \frac{1-\alpha(x)}{\alpha(x)} \boldsymbol{v}_{1} \cdot \boldsymbol{u}_{2} d \sigma \\
& +\left(\boldsymbol{A} \boldsymbol{u}_{1}, \boldsymbol{v}_{2}\right)_{L^{2}\left(\Omega, \mathbf{R}^{n}\right)} .
\end{aligned}
$$

However, we have, by Green's formula,

$$
\begin{aligned}
& \left(\boldsymbol{\mathfrak { A }} \boldsymbol{u}_{1}, \boldsymbol{v}_{2}\right)_{\boldsymbol{L}^{2}\left(\Omega, \mathbf{R}^{n}\right)} \\
= & -\int_{\Omega} \boldsymbol{\nabla} \boldsymbol{u}_{1} \cdot \mathbf{a}(x) \cdot \boldsymbol{\nabla} \boldsymbol{v}_{2} d x-\int_{\{\alpha(x) \neq 0\}} \frac{1-\alpha(x)}{\alpha(x)} \boldsymbol{v}_{2} \cdot \boldsymbol{u}_{1} d \sigma .
\end{aligned}
$$

Therefore, we obtain from formulas (5.10) and (5.11) that

$$
\begin{aligned}
(\boldsymbol{A U}, \boldsymbol{V})_{\mathcal{X}}= & \int_{\Omega} \boldsymbol{\nabla} \boldsymbol{v}_{1} \cdot \mathbf{a}(x) \cdot \boldsymbol{\nabla} \boldsymbol{u}_{2} d x+\int_{\{\alpha(x) \neq 0\}} \frac{1-\alpha(x)}{\alpha(x)} \boldsymbol{v}_{1} \cdot \boldsymbol{u}_{2} d \sigma \\
& -\int_{\Omega} \boldsymbol{\nabla} \boldsymbol{u}_{1} \cdot \mathbf{a}(x) \cdot \boldsymbol{\nabla} \boldsymbol{v}_{2} d x-\int_{\{\alpha(x) \neq 0\}} \frac{1-\alpha(x)}{\alpha(x)} \boldsymbol{v}_{2} \cdot \boldsymbol{u}_{1} d \sigma
\end{aligned}
$$

Similarly, we have the formula

$$
\begin{aligned}
-(\boldsymbol{U}, \boldsymbol{A} \boldsymbol{V})_{\mathcal{X}}= & -\int_{\Omega} \boldsymbol{\nabla} \boldsymbol{u}_{1} \cdot \mathbf{a}(x) \cdot \boldsymbol{\nabla} \boldsymbol{v}_{2} d x-\int_{\{\alpha(x) \neq 0\}} \frac{1-\alpha(x)}{\alpha(x)} \boldsymbol{u}_{1} \cdot \boldsymbol{v}_{2} d \sigma \\
& +\int_{\Omega} \boldsymbol{\nabla} \boldsymbol{v}_{1} \cdot \mathbf{a}(x) \cdot \boldsymbol{\nabla} \boldsymbol{u}_{2} d x+\int_{\{\alpha(x) \neq 0\}} \frac{1-\alpha(x)}{\alpha(x)} \boldsymbol{u}_{2} \cdot \boldsymbol{v}_{1} d \sigma
\end{aligned}
$$

The desired formula (5.8) follows from formulas (5.12) and (5.13).

Step 5: If $\lambda \neq 0$, we show that

$$
\|(\lambda \boldsymbol{I}-\boldsymbol{A}) \boldsymbol{U}\|_{\mathcal{X}} \geq|\lambda|\|\boldsymbol{U}\|_{\mathcal{X}} \text { for all } \boldsymbol{U} \in D(\boldsymbol{A}) .
$$

In other words, we show that the resolvent $(\lambda \boldsymbol{I}-\boldsymbol{A})^{-1}$ exists and satisfies the condition

$$
\left\|(\lambda \boldsymbol{I}-\boldsymbol{A})^{-1}\right\| \leq \frac{1}{|\lambda|} \quad \text { for every } \lambda \neq 0,
$$

since $\lambda \boldsymbol{I}-\boldsymbol{A}$ is surjective for every $\lambda \neq 0$ (see Step 3 ).

Indeed, we have, by formula (5.9),

$$
\begin{aligned}
\|(\lambda \boldsymbol{I}-\boldsymbol{A}) \boldsymbol{U}\|_{\mathcal{X}}^{2} & =((\lambda \boldsymbol{I}-\boldsymbol{A}) \boldsymbol{U},(\lambda \boldsymbol{I}-\boldsymbol{A}) \boldsymbol{U})_{\mathcal{X}} \\
& =\lambda^{2}\|\boldsymbol{U}\|_{\mathcal{X}}^{2}-\lambda\left\{(\boldsymbol{A} \boldsymbol{U}, \boldsymbol{U})_{\mathcal{X}}+(\boldsymbol{U}, \boldsymbol{A U})_{\mathcal{X}}\right\}+\|\boldsymbol{A} \boldsymbol{U}\|_{\mathcal{X}}^{2}
\end{aligned}
$$




$$
\begin{aligned}
& =\lambda^{2}\|\boldsymbol{U}\|_{\mathcal{X}}^{2}+\|\boldsymbol{A} \boldsymbol{U}\|_{\mathcal{X}}^{2} \\
& \geq \lambda^{2}\|\boldsymbol{U}\|_{\mathcal{X}}^{2} \quad \text { for all } \boldsymbol{U} \in D(\boldsymbol{A}) .
\end{aligned}
$$

This proves the desired inequality (5.14).

Step 6: Therefore, by applying Theorem 5.2 to our situation ([25, Chapter IX, Section 9, Remark]) we obtain that the operator $\boldsymbol{A}$ generates a strongly continuous group $e^{t \boldsymbol{A}}$ of unitary operators in the Hilbert space $\boldsymbol{X}$.

Step 7: Finally, the desired representation formula (5.3) is an immediate consequence of [24, Theorem 1] and [8, Chapter II, Theorem 7.4].

Now the proof of Theorem 5.1 is complete.

By applying Theorem 5.1 to the initial-boundary value problem (1.1), we can obtain the representation formula (2.2) of a (unique) solution of problem (1.1) (cf. [7, Theorem 2.1]):

Theorem 5.3 Let $\boldsymbol{u}(t)$ be a (unique) solution of the initial-boundary value problem (1.1) with data

$$
\boldsymbol{u}_{0} \in D(\mathfrak{A}), \boldsymbol{u}_{1} \in \mathcal{H}, \boldsymbol{f}(t) \in C([0, \infty), D(\mathfrak{A})) .
$$

Then it can be represented explicitly as formula (2.2).

Proof Since we have the formula

$$
\boldsymbol{U}(t)=e^{t \boldsymbol{A}} \boldsymbol{U}_{0}+\int_{0}^{t} e^{(t-s) \boldsymbol{A}} \boldsymbol{F}(s) d s,
$$

it follows from formula (5.3) that

$$
\begin{aligned}
& \left(\begin{array}{c}
\boldsymbol{u}(t) \\
\boldsymbol{u}^{\prime}(t)
\end{array}\right) \\
& =\left(\cos (t \mathfrak{C})\left(\begin{array}{ll}
\boldsymbol{I} & \mathbf{0} \\
\mathbf{0} & \boldsymbol{I}
\end{array}\right)+\mathfrak{C}^{-1} \sin (t \mathfrak{C})\left(\begin{array}{cc}
\mathbf{0} & \boldsymbol{I} \\
\mathfrak{A} & 0
\end{array}\right)\right)\left(\begin{array}{l}
\boldsymbol{u}_{0} \\
\boldsymbol{u}_{1}
\end{array}\right) \\
& +\int_{0}^{t}\left(\cos ((t-s) \mathfrak{C})\left(\begin{array}{ll}
\boldsymbol{I} & 0 \\
0 & \boldsymbol{I}
\end{array}\right)+\mathfrak{C}^{-1} \sin ((t-s) \mathfrak{C})\left(\begin{array}{cc}
\mathbf{0} & \boldsymbol{I} \\
\mathfrak{A} & 0
\end{array}\right)\right)\left(\begin{array}{c}
0 \\
\boldsymbol{f}(s)
\end{array}\right) d s \\
& =\cos (t \mathfrak{C})\left(\begin{array}{l}
\boldsymbol{u}_{0} \\
\boldsymbol{u}_{1}
\end{array}\right)+\mathfrak{C}^{-1} \sin (t \mathfrak{C})\left(\begin{array}{c}
\boldsymbol{u}_{1} \\
\mathfrak{A} \boldsymbol{u}_{0}
\end{array}\right) \\
& +\int_{0}^{t} \cos ((t-s) \mathfrak{C})\left(\begin{array}{c}
0 \\
\boldsymbol{f}(s)
\end{array}\right) d s+\int_{0}^{t} \mathfrak{C}^{-1} \sin ((t-s) \mathfrak{C})\left(\begin{array}{c}
\boldsymbol{f}(s) \\
0
\end{array}\right) d s .
\end{aligned}
$$

This proves the desired formula (2.2).

The proof of Theorem 5.3 is complete.

\section{Existence and Uniqueness Theorem for Problem (1.1)}

In this section we prove the fundamental existence and uniqueness theorem for the initial-boundary value problem (1.1) (Theorem 6.1).

First, we have the following existence and uniqueness theorem for the initial value problem (5.2) (cf. [13], [3, Chapitre 6, Théorème 6.9]): 
Proposition 6.1 For any given data

$$
\boldsymbol{U}_{0} \in D(\boldsymbol{A}), \boldsymbol{F}(t) \in C([0, \infty), D(\boldsymbol{A})),
$$

the initial value problem (5.2) has a unique solution

$$
\boldsymbol{U}(t) \in C^{1}([0, \infty), \boldsymbol{\mathcal { X }}) \cap C([0, \infty), D(\boldsymbol{A})) .
$$

Moreover, we have the energy inequality

$$
\|\boldsymbol{U}(t)\|_{\mathcal{X}} \leq\|\boldsymbol{U}(0)\|_{\mathcal{X}}+\int_{0}^{t}\|\boldsymbol{F}(s)\|_{\mathcal{X}} d s
$$

Here we recall that

$$
\|\boldsymbol{U}\|_{\mathcal{X}}=\left\|\left(\begin{array}{l}
\boldsymbol{u} \\
\boldsymbol{v}
\end{array}\right)\right\|_{\mathcal{X}}=\|\boldsymbol{u}\|_{\mathcal{H}}+\|\boldsymbol{v}\|_{\boldsymbol{L}^{2}\left(\Omega, \mathbf{R}^{n}\right)}
$$

Indeed, the desired energy inequality (6.1) follows from formula (5.15), since $e^{t \boldsymbol{A}}$ is a contraction group in $\boldsymbol{X}$.

Therefore, by applying Proposition 6.1 to the initial-boundary value problem (1.1) we have the following existence and uniqueness theorem for linear elastodynamics (cf. [16, Proposition 3.6, Part (i)]):

Theorem 6.1 For any given data

$$
\boldsymbol{u}_{0} \in D(\boldsymbol{\mathfrak { A }}), \boldsymbol{u}_{1} \in \mathcal{H}, \boldsymbol{f}(t) \in C([0, \infty), D(\boldsymbol{\mathfrak { A }})),
$$

the initial-boundary value problem (1.1) has a unique solution

$$
\boldsymbol{u}(t) \in C^{1}([0, \infty), \mathcal{H}) \cap C([0, \infty), D(\mathfrak{A}))
$$

Moreover, we have the energy inequality

$$
\begin{aligned}
& \|\boldsymbol{u}(t)\|_{\mathcal{H}}+\left\|\boldsymbol{u}^{\prime}(t)\right\|_{\boldsymbol{L}^{2}\left(\Omega, \mathbf{R}^{n}\right)} \\
\leq & \left\|\boldsymbol{u}_{0}\right\|_{\mathcal{H}}+\left\|\boldsymbol{u}_{1}\right\|_{L^{2}\left(\Omega, \mathbf{R}^{n}\right)}+\int_{0}^{t}\|\boldsymbol{f}(s)\|_{\boldsymbol{L}^{2}\left(\Omega, \mathbf{R}^{n}\right)} d s .
\end{aligned}
$$

Indeed, the energy inequality (6.3) follows from inequality (6.1) and formula $(6.2)$.

\section{Energy Estimates for Problem (1.1)}

In this section we prove the fundamental energy inequality (2.3) for the initialboundary value problem (1.1) (Theorem 7.1).

First, we prove the following energy inequality for the initial value problem $(5.2)$ : 
Proposition 7.1 Assume that a function

$$
\boldsymbol{U}(t) \in C^{1}([0, \infty), \boldsymbol{X}) \cap C([0, \infty), D(\boldsymbol{A}))
$$

is a (unique) solution of problem (5.2) with data

$$
\boldsymbol{U}_{0} \in D(\boldsymbol{A}), \boldsymbol{F}(t) \in C^{1}([0, \infty), \boldsymbol{X})
$$

Then we have the energy inequality

$$
\left\|\boldsymbol{U}^{\prime}(t)\right\|_{\mathcal{X}} \leq\left\|\boldsymbol{A} \boldsymbol{U}_{0}+\boldsymbol{F}(0)\right\|_{\mathcal{X}}+\int_{0}^{t}\left\|\boldsymbol{F}^{\prime}(s)\right\|_{\mathcal{X}} d s .
$$

Proof By formula (5.15), it follows that

$$
\begin{aligned}
\boldsymbol{U}(t) & =e^{t \boldsymbol{A}} \boldsymbol{U}_{0}+\int_{0}^{t} e^{(t-s) \boldsymbol{A}} \boldsymbol{F}(s) d s \\
& =e^{t \boldsymbol{A}} \boldsymbol{U}_{0}+\int_{0}^{t} e^{s \boldsymbol{A}} \boldsymbol{F}(t-s) d s .
\end{aligned}
$$

By differentiating formula (7.3), we obtain that

$$
\begin{aligned}
\boldsymbol{U}^{\prime}(t) & =e^{t \boldsymbol{A}}\left(\boldsymbol{A} \boldsymbol{U}_{0}\right)+e^{t \boldsymbol{A}} \boldsymbol{F}(0)+\int_{0}^{t} e^{s \boldsymbol{A}} \boldsymbol{F}^{\prime}(t-s) d s \\
& =e^{t \boldsymbol{A}}\left(\boldsymbol{A} \boldsymbol{U}_{0}+\boldsymbol{F}(0)\right)+\int_{0}^{t} e^{s \boldsymbol{A}} \boldsymbol{F}^{\prime}(t-s) d s
\end{aligned}
$$

Since $e^{t \boldsymbol{A}}$ is a contraction group in $\boldsymbol{\mathcal { X }}$, it follows from formula (7.4) that

$$
\left\|\boldsymbol{U}^{\prime}(t)\right\|_{\mathcal{X}} \leq\left\|\boldsymbol{A} \boldsymbol{U}_{0}+\boldsymbol{F}(0)\right\|_{\mathcal{X}}+\int_{0}^{t}\left\|\boldsymbol{F}^{\prime}(t-s)\right\| \mathcal{X} d s
$$

However, we have the formula

$$
\int_{0}^{t}\left\|\boldsymbol{F}^{\prime}(t-s)\right\|_{\mathcal{X}} d s=-\int_{t}^{0}\left\|\boldsymbol{F}^{\prime}(\tau)\right\|_{\mathcal{X}} d \tau=\int_{0}^{t}\left\|\boldsymbol{F}^{\prime}(\tau)\right\|_{\mathcal{X}} d \tau .
$$

Therefore, the desired energy inequality (7.2) follows by combining inequality (7.5) and formula (7.6).

The proof of Proposition 7.1 is complete.

By applying Proposition 7.1 to the initial-boundary value problem (1.1), we have the following energy inequality for linear elastodynamics (cf. [15, Proposition 3.9], [16, Proposition 3.6, Part (ii)]):

Theorem 7.1 Assume that a function

$$
\left.\boldsymbol{u}(t) \in C^{2}\left([0, \infty), \boldsymbol{L}^{2}\left(\Omega, \mathbf{R}^{n}\right)\right) \cap C^{1}([0, \infty), \boldsymbol{H})\right) \cap C([0, \infty), D(\boldsymbol{A}))
$$

is a (unique) solution of the initial-boundary value problem (1.1) with data

$$
\begin{aligned}
& \boldsymbol{u}_{0} \in D(\boldsymbol{\mathfrak { A }}), \boldsymbol{u}_{1} \in \mathcal{H}, \\
& \boldsymbol{f}(t) \in C^{1}\left([0, \infty), \boldsymbol{L}^{2}\left(\Omega, \mathbf{R}^{n}\right)\right) .
\end{aligned}
$$

Then we have the energy inequality (2.3). 
Proof First, we have the formulas

$$
\left\|\boldsymbol{U}^{\prime}(t)\right\|_{\mathcal{X}}=\left\|\left(\begin{array}{c}
\boldsymbol{u}^{\prime}(t) \\
\boldsymbol{u}^{\prime \prime}(t)
\end{array}\right)\right\|_{\mathcal{X}}=\left\|\boldsymbol{u}^{\prime}(t)\right\|_{\mathcal{H}}+\left\|\boldsymbol{u}^{\prime \prime}(t)\right\|_{\boldsymbol{L}^{2}\left(\Omega, \mathbf{R}^{n}\right)}
$$

and

$$
\int_{0}^{t}\left\|\boldsymbol{F}^{\prime}(s)\right\| \boldsymbol{\mathcal { X }} d s=\int_{0}^{t}\left\|\boldsymbol{f}^{\prime}(s)\right\|_{\boldsymbol{L}^{2}\left(\Omega, \mathbf{R}^{n}\right)} d s .
$$

Moreover, we have, by inequalities (5.4),

$$
\begin{aligned}
C_{1}\left\|\boldsymbol{u}_{0}\right\|_{\boldsymbol{H}^{2}\left(\Omega, \mathbf{R}^{n}\right)} & \leq\left\|\boldsymbol{A} \boldsymbol{u}_{0}\right\|_{\boldsymbol{L}^{2}\left(\Omega, \mathbf{R}^{n}\right)} \\
& \leq C_{2}\left\|\boldsymbol{u}_{0}\right\|_{\boldsymbol{H}^{2}\left(\Omega, \mathbf{R}^{n}\right)} \quad \text { for all } \boldsymbol{u}_{0} \in D(\boldsymbol{A}) .
\end{aligned}
$$

Hence we have the inequality

$$
\begin{aligned}
\left\|\boldsymbol{A} \boldsymbol{U}_{0}+\boldsymbol{F}(0)\right\|_{\mathcal{X}} & =\left\|\left(\begin{array}{c}
\boldsymbol{u}_{1} \\
\boldsymbol{\mathfrak { A }} \boldsymbol{u}_{0}+\boldsymbol{f}(0)
\end{array}\right)\right\|_{\mathcal{X}} \\
& =\left\|\boldsymbol{u}_{1}\right\|_{\mathcal{H}}+\left\|\boldsymbol{A} \boldsymbol{u}_{0}+\boldsymbol{f}(0)\right\|_{\boldsymbol{L}^{2}\left(\Omega, \mathbf{R}^{n}\right)} \\
& \leq\left\|\boldsymbol{u}_{1}\right\|_{\mathcal{H}}+C_{2}\left\|\boldsymbol{u}_{0}\right\|_{\boldsymbol{H}^{2}\left(\Omega, \mathbf{R}^{n}\right)}+\|\boldsymbol{f}(0)\|_{\boldsymbol{L}^{2}\left(\Omega, \mathbf{R}^{n}\right)}
\end{aligned}
$$

Therefore, by using formulas (7.8) and (7.9) and inequality (7.11) we obtain from the energy inequality (7.2) that

$$
\begin{aligned}
& \left\|\boldsymbol{u}^{\prime}(t)\right\|_{\mathcal{H}}+\left\|\boldsymbol{u}^{\prime \prime}(t)\right\|_{\boldsymbol{L}^{2}\left(\Omega, \mathbf{R}^{n}\right)} \\
\leq & C_{2}\left\|\boldsymbol{u}_{0}\right\|_{\boldsymbol{H}^{2}\left(\Omega, \mathbf{R}^{n}\right)}+\left\|\boldsymbol{u}_{1}\right\|_{\mathcal{H}}+\|\boldsymbol{f}(0)\|_{\boldsymbol{L}^{2}\left(\Omega, \mathbf{R}^{n}\right)}+\int_{0}^{t}\left\|\boldsymbol{f}^{\prime}(s)\right\|_{\boldsymbol{L}^{2}\left(\Omega, \mathbf{R}^{n}\right)} d s .
\end{aligned}
$$

On the other hand, since we have the equation

$$
\boldsymbol{u}^{\prime \prime}(t)=\boldsymbol{\mathfrak { A }} \boldsymbol{u}(t)+\boldsymbol{f}(t)
$$

it follows from inequalities (7.10) with $\boldsymbol{u}_{0}:=\boldsymbol{u}(t)$ that

$$
\begin{aligned}
& C_{1}\|\boldsymbol{u}(t)\|_{\boldsymbol{H}^{2}\left(\Omega, \mathbf{R}^{n}\right)} \leq\|\boldsymbol{A} \boldsymbol{u}(t)\|_{\boldsymbol{L}^{2}\left(\Omega, \mathbf{R}^{n}\right)} \\
& \leq\left\|\boldsymbol{u}^{\prime \prime}(t)-\boldsymbol{f}(t)\right\|_{\boldsymbol{L}^{2}\left(\Omega, \mathbf{R}^{n}\right)} \\
& \leq\left\|\boldsymbol{u}^{\prime \prime}(t)\right\|_{\boldsymbol{L}^{2}\left(\Omega, \mathbf{R}^{n}\right)}+\|\boldsymbol{f}(t)\|_{\boldsymbol{L}^{2}\left(\Omega, \mathbf{R}^{n}\right)} .
\end{aligned}
$$

Moreover, since we have the fundamental formula of calculus

$$
\boldsymbol{f}(t)=\boldsymbol{f}(0)+\int_{0}^{t} \boldsymbol{f}^{\prime}(s) d s
$$

it follows that

$$
\|\boldsymbol{f}(t)\|_{\boldsymbol{L}^{2}\left(\Omega, \mathbf{R}^{n}\right)} \leq\|\boldsymbol{f}(0)\|_{\boldsymbol{L}^{2}\left(\Omega, \mathbf{R}^{n}\right)}+\int_{0}^{t}\left\|\boldsymbol{f}^{\prime}(s)\right\|_{\boldsymbol{L}^{2}\left(\Omega, \mathbf{R}^{n}\right)} d s .
$$

Therefore, by combining inequalities (7.13) and (7.14) we obtain that

$$
\begin{aligned}
& \|\boldsymbol{u}(t)\|_{\boldsymbol{H}^{2}\left(\Omega, \mathbf{R}^{n}\right)} \\
\leq & \frac{1}{C_{1}}\left(\left\|\boldsymbol{u}^{\prime \prime}(t)\right\|_{\boldsymbol{L}^{2}\left(\Omega, \mathbf{R}^{n}\right)}+\|\boldsymbol{f}(0)\|_{\boldsymbol{L}^{2}\left(\Omega, \mathbf{R}^{n}\right)}+\int_{0}^{t}\left\|\boldsymbol{f}^{\prime}(s)\right\|_{\boldsymbol{L}^{2}\left(\Omega, \mathbf{R}^{n}\right)} d s\right) .
\end{aligned}
$$

The desired energy inequality (2.3) follows by combining inequalities (7.12) and (7.15).

The proof of Theorem 7.1 is complete. 


\section{Regularity Theorem for Problem (1.1)}

In this section we prove the following regularity theorem for the initial-boundary value problem (1.1) (cf. [15, Theorem 4.1], [16, Proposition 3.7]):

Theorem 8.1 Assume that conditions (T) and (A) are satisfied. If a function

$$
\boldsymbol{u}(t) \in C^{1}\left([0, \infty), \boldsymbol{L}^{2}\left(\Omega, \mathbf{R}^{n}\right)\right) \cap C([0, \infty), \boldsymbol{H})
$$

is a solution of the initial-boundary value problem (1.1) with data

$$
\boldsymbol{u}_{0} \in D(\boldsymbol{\mathfrak { A }}), \boldsymbol{u}_{1} \in \mathcal{H}, \boldsymbol{f}(t) \in C^{1}\left([0, \infty), \boldsymbol{L}^{2}\left(\Omega, \mathbf{R}^{n}\right)\right),
$$

then the solution $\boldsymbol{u}(t)$ enjoys the regularity property (2.1).

Proof The proof of Theorem 8.1 is divided into four steps.

Step 1: We construct a sequence

$$
\left\{\boldsymbol{f}_{j}(t)\right\} \subset C^{1}\left([0, \infty), \boldsymbol{L}^{2}\left(\Omega, \mathbf{R}^{n}\right)\right) \cap C([0, \infty), D(\mathfrak{A})),
$$

such that

$$
\begin{aligned}
& \boldsymbol{f}_{j}(\cdot, t) \longrightarrow \boldsymbol{f}(\cdot, t) \quad \text { in } \boldsymbol{L}^{2}\left(\Omega, \mathbf{R}^{n}\right) \text { for every } t \in \mathbf{R} \text { as } j \rightarrow \infty \\
& \boldsymbol{f}_{j}^{\prime}(\cdot, t) \longrightarrow \boldsymbol{f}^{\prime}(\cdot, t) \quad \text { in } \boldsymbol{L}^{2}\left(\Omega, \mathbf{R}^{n}\right) \text { for every } t \in \mathbf{R} \text { as } j \rightarrow \infty
\end{aligned}
$$

We recall (Theorem 2.2) that the operator $\mathfrak{A}$ generates an analytic semigroup $e^{t \mathfrak{A}}$ in some sector containing the positive real axis.

For every $\varepsilon>0$, we let

$$
M_{\varepsilon} \boldsymbol{f}(x, t)=\frac{1}{\varepsilon} \int_{0}^{\varepsilon} e^{s \mathfrak{A}} \boldsymbol{f}(x, t) d s .
$$

Step 1-1: First, we show that

$$
M_{\varepsilon} \boldsymbol{f}(\cdot, t) \in C([0, \infty), D(\mathfrak{A})) .
$$

Indeed, we have, for any $0<h<\varepsilon$,

$$
\begin{aligned}
e^{h \mathfrak{A}}\left(M_{\varepsilon} \boldsymbol{f}(x, t)\right) & =\frac{1}{\varepsilon} \int_{0}^{\varepsilon} e^{h \mathfrak{A}}\left(e^{s \mathfrak{A}} \boldsymbol{f}(\cdot, t)\right) d s=\frac{1}{\varepsilon} \int_{0}^{\varepsilon} e^{(h+s) \mathfrak{A}} \boldsymbol{f}(\cdot, t) d s \\
& =\frac{1}{\varepsilon} \int_{h}^{\varepsilon+h} e^{s \mathfrak{A}} \boldsymbol{f}(\cdot, t) d s .
\end{aligned}
$$

Hence it follows that

$$
\begin{aligned}
\left(\frac{e^{h \mathfrak{A}}-\boldsymbol{I}}{h}\right) M_{\varepsilon} \boldsymbol{f}(x, t) & =\frac{1}{\varepsilon}\left(\frac{1}{h} \int_{h}^{\varepsilon+h} e^{s \mathfrak{A}} \boldsymbol{f}(\cdot, t) d s-\frac{1}{h} \int_{0}^{\varepsilon} e^{s \mathfrak{A}} \boldsymbol{f}(\cdot, t) d s\right) \\
& =\frac{1}{\varepsilon}\left(\frac{1}{h} \int_{\varepsilon}^{\varepsilon+h} e^{s \mathfrak{A}} \boldsymbol{f}(\cdot, t) d s-\frac{1}{h} \int_{0}^{h} e^{s \mathfrak{A}} \boldsymbol{f}(\cdot, t) d s\right) .
\end{aligned}
$$

However, we have, as $h \downarrow 0$,

$$
\frac{1}{h} \int_{\varepsilon}^{\varepsilon+h} e^{s \mathfrak{A}} \boldsymbol{f}(\cdot, t) d s \longrightarrow e^{\varepsilon \mathfrak{A}} \boldsymbol{f}(\cdot, t) \quad \text { in } \boldsymbol{L}^{2}\left(\Omega, \mathbf{R}^{n}\right),
$$




$$
\frac{1}{h} \int_{0}^{h} e^{s \mathfrak{A}} \boldsymbol{f}(\cdot, t) d s \longrightarrow \boldsymbol{f}(\cdot, t) \quad \text { in } \boldsymbol{L}^{2}\left(\Omega, \mathbf{R}^{n}\right),
$$

since the integrand $e^{s \mathfrak{A}} \boldsymbol{f}(\cdot, t)$ is strongly continuous in $s$.

Therefore, we find from formula (8.4) that, as $h \downarrow 0$,

$$
\left(\frac{e^{h \mathfrak{A}}-\boldsymbol{I}}{h}\right) M_{\varepsilon} \boldsymbol{f}(x, t) \longrightarrow \frac{1}{\varepsilon}\left(e^{\varepsilon \mathfrak{A}} \boldsymbol{f}(\cdot, t)-\boldsymbol{f}(\cdot, t)\right) .
$$

This proves that

$$
\left\{\begin{array}{l}
M_{\varepsilon} \boldsymbol{f}(\cdot, t) \in D(\mathfrak{A}), \\
\mathfrak{A} M_{\varepsilon} \boldsymbol{f}(\cdot, t)=\frac{1}{\varepsilon}\left(e^{\varepsilon \mathfrak{A}} \boldsymbol{f}(\cdot, t)-\boldsymbol{f}(\cdot, t)\right) .
\end{array}\right.
$$

Since $\boldsymbol{f}(\cdot, t) \in C\left([0, \infty), \boldsymbol{L}^{2}\left(\Omega, \mathbf{R}^{n}\right)\right)$, it follows from formula (8.5) that

$$
\begin{aligned}
& \boldsymbol{A}\left(M_{\varepsilon} \boldsymbol{f}\right)(\cdot, t)=\frac{1}{\varepsilon}\left(e^{\varepsilon \mathfrak{A}}-I\right) \boldsymbol{f}(\cdot, t) \\
\longrightarrow & \frac{1}{\varepsilon}\left(e^{\varepsilon \mathfrak{A}}-I\right) \boldsymbol{f}\left(\cdot, t^{\prime}\right)=\boldsymbol{A}\left(M_{\varepsilon} \boldsymbol{f}\right)\left(\cdot, t^{\prime}\right) \quad \text { in } \boldsymbol{L}^{2}\left(\Omega, \mathbf{R}^{n}\right) \text { as } t \rightarrow t^{\prime} .
\end{aligned}
$$

In view of inequalities (5.4), this proves that

$$
M_{\varepsilon} \boldsymbol{f}(\cdot, t) \longrightarrow M_{\varepsilon} \boldsymbol{f}\left(\cdot, t^{\prime}\right) \quad \text { in the domain } D(\mathfrak{A}) \text { as } t \rightarrow t^{\prime} .
$$

Therefore, we have proved the desired assertion (8.3).

Step 1-2: Secondly, we show that

$$
M_{\varepsilon} \boldsymbol{f}(\cdot, t) \in C^{1}\left([0, \infty), \boldsymbol{L}^{2}\left(\Omega, \mathbf{R}^{n}\right)\right) .
$$

Indeed, since $\boldsymbol{f}(\cdot, t) \in C^{1}\left([0, \infty), \boldsymbol{L}^{2}\left(\Omega, \mathbf{R}^{n}\right)\right)$, we have the formula

$$
\frac{d}{d t}\left(M_{\varepsilon} \boldsymbol{f}(x, t)\right)=\frac{1}{\varepsilon} \int_{0}^{\varepsilon} e^{s \mathfrak{A}} \frac{\partial \boldsymbol{f}}{\partial t}(x, t) d s .
$$

Moreover, since the semigroup $e^{s \mathfrak{A}}$ is analytic, it follows that we have, as $t \rightarrow t^{\prime}$,

$$
\begin{aligned}
& M_{\varepsilon} \boldsymbol{f}(\cdot, t)=\frac{1}{\varepsilon} \int_{0}^{\varepsilon} e^{s \mathfrak{A}} \boldsymbol{f}(\cdot, t) d s \\
& \longrightarrow \frac{1}{\varepsilon} \int_{0}^{\varepsilon} e^{s \mathfrak{A}} \boldsymbol{f}\left(\cdot, t^{\prime}\right) d s=M_{\varepsilon} \boldsymbol{f}\left(\cdot, t^{\prime}\right) \quad \text { in } \boldsymbol{L}^{2}\left(\Omega, \mathbf{R}^{n}\right)
\end{aligned}
$$

and

$$
\begin{aligned}
& \frac{d}{d t}\left(M_{\varepsilon} \boldsymbol{f}(\cdot, t)\right)=\frac{1}{\varepsilon} \int_{0}^{\varepsilon} e^{s \mathfrak{A}} \frac{\partial \boldsymbol{f}}{\partial t}(\cdot, t) d s \\
& \longrightarrow \frac{1}{\varepsilon} \int_{0}^{\varepsilon} e^{s \mathfrak{A}} \frac{\partial \boldsymbol{f}}{\partial t}\left(\cdot, t^{\prime}\right) d s=\frac{d}{d t}\left(M_{\varepsilon} \boldsymbol{f}\left(\cdot, t^{\prime}\right)\right) \quad \text { in } \boldsymbol{L}^{2}\left(\Omega, \mathbf{R}^{n}\right) .
\end{aligned}
$$

Therefore, we have proved the desired assertion (8.6).

Step 1-3: Thirdly, we have, as $\varepsilon \downarrow 0$,

$$
M_{\varepsilon} \boldsymbol{f}(\cdot, t)=\frac{1}{\varepsilon} \int_{0}^{\varepsilon} e^{s \mathfrak{A}} \boldsymbol{f}(\cdot, t) d s
$$




$$
\begin{aligned}
& \left.\longrightarrow \boldsymbol{f}(\cdot, t) \quad \text { in } \boldsymbol{L}^{2}\left(\Omega, \mathbf{R}^{n}\right)\right) \text { for every } t \in \mathbf{R}, \\
& \frac{d}{d t}\left(M_{\varepsilon} \boldsymbol{f}(\cdot, t)\right)=\frac{1}{\varepsilon} \int_{0}^{\varepsilon} e^{s \mathfrak{A}} \frac{\partial \boldsymbol{f}}{\partial t}(\cdot, t) d s \\
& \left.\longrightarrow \frac{\partial \boldsymbol{f}}{\partial t}(\cdot, t) \quad \text { in } \boldsymbol{L}^{2}\left(\Omega, \mathbf{R}^{n}\right)\right) \text { for every } t \in \mathbf{R},
\end{aligned}
$$

since the functions $e^{s \mathfrak{A}} \boldsymbol{f}(\cdot, t)$ and $e^{s \mathfrak{A}}(\partial \boldsymbol{f})(\partial t)(\cdot, t)$ are strongly continuous in $s$.

Therefore, if we let

$$
\boldsymbol{f}_{j}(x, t)=M_{1 / j} \boldsymbol{f}(\cdot, t), \quad j=1,2, \ldots,
$$

then we find from assertions (8.3), (8.6) and (8.7) that the sequence $\left\{\boldsymbol{f}_{j}(t)\right\}$ satisfies the desired conditions (8.1) and (8.2).

Step 2: We let

$$
\boldsymbol{F}_{j}(t)=\left(\begin{array}{c}
\mathbf{0} \\
\boldsymbol{f}_{j}(t)
\end{array}\right)
$$

and consider the Cauchy problem

$$
\left\{\begin{array}{l}
\boldsymbol{U}^{\prime}(t)=\boldsymbol{A U}(t)+\boldsymbol{F}_{j}(t), \quad t>0 \\
\boldsymbol{U}(0)=\boldsymbol{U}_{0}
\end{array}\right.
$$

Here recall that

$$
\boldsymbol{X}=\boldsymbol{H} \times \boldsymbol{L}^{2}\left(\Omega, \mathbf{R}^{n}\right),
$$

and

$$
A=\left(\begin{array}{cc}
\mathbf{0} & \boldsymbol{I} \\
\mathfrak{A} & 0
\end{array}\right): \mathcal{X} \longrightarrow \mathcal{X}
$$

with domain

$$
D(\boldsymbol{A})=D(\mathfrak{A}) \times \mathcal{H} .
$$

By condition (8.1), we have the following three assertions:

(a) $\boldsymbol{F}_{j} \in D(\boldsymbol{A})$.

(b) $\boldsymbol{F}_{j}(t) \in C([0, \infty), \boldsymbol{X})$.

(c) $\boldsymbol{A F}_{j}(t) \in C([0, \infty), \boldsymbol{\mathcal { X }})$.

Therefore, by applying the Hille-Yosida theorem ([8, Chapter II, Theorem 1.3]) we can find solutions

$$
\boldsymbol{U}_{j}(t)=\left(\begin{array}{l}
\boldsymbol{u}_{j}(t) \\
\boldsymbol{v}_{j}(t)
\end{array}\right) \in C^{1}([0, \infty), \boldsymbol{X}) \cap C([0, \infty), D(\boldsymbol{A}))
$$

of problem (8.8). In particular, we have the formulas

$$
\left(\begin{array}{l}
\frac{d \boldsymbol{u}_{j}}{d t} \\
\frac{d \boldsymbol{v}_{j}}{d t}
\end{array}\right)=\left(\begin{array}{cc}
\mathbf{0} & \boldsymbol{I} \\
\mathfrak{A} & 0
\end{array}\right)\left(\begin{array}{c}
\boldsymbol{u}_{j}(t) \\
\boldsymbol{v}_{j}(t)
\end{array}\right)+\left(\begin{array}{c}
\mathbf{0} \\
\boldsymbol{f}_{j}(t)
\end{array}\right)=\left(\begin{array}{c}
\boldsymbol{v}_{j}(t) \\
\boldsymbol{A} \boldsymbol{u}_{j}(t)+\boldsymbol{f}_{j}(t)
\end{array}\right) .
$$

By combining assertion (8.9) and formulas (8.10), we obtain the following four assertions:

(i) $\boldsymbol{u}_{j} \in C^{1}([0, \infty), \mathcal{H})$.

(ii) $\frac{d \boldsymbol{u}_{j}}{d t}=\boldsymbol{v}_{j} \in C^{1}\left([0, \infty), \boldsymbol{L}^{2}\left(\Omega, \mathbf{R}^{n}\right)\right)$. 
(iii) $\frac{d^{2} \boldsymbol{u}_{j}}{d t^{2}}=\frac{d \boldsymbol{v}_{j}}{d t} \in C\left([0, \infty), \boldsymbol{L}^{2}\left(\Omega, \mathbf{R}^{n}\right)\right)$.

(iv) $\boldsymbol{A} \boldsymbol{u}_{j}=\frac{d \boldsymbol{v}_{j}}{d t}-\boldsymbol{f}_{j} \in C\left([0, \infty), \boldsymbol{L}^{2}\left(\Omega, \mathbf{R}^{n}\right)\right)$.

Since $\boldsymbol{A} \boldsymbol{u}_{j}(t) \in C\left([0, \infty), \boldsymbol{L}^{2}\left(\Omega, \mathbf{R}^{n}\right)\right)$, it follows from inequalities (7.10) with $\boldsymbol{u}_{0}:=$ $\boldsymbol{u}_{j}(t)-\boldsymbol{u}_{j}\left(t^{\prime}\right)$ that we have, as $t \rightarrow t^{\prime}$,

$$
\begin{aligned}
\left\|\boldsymbol{u}_{j}(t)-\boldsymbol{u}_{j}\left(t^{\prime}\right)\right\|_{D(\mathfrak{A})} & =\left\|\boldsymbol{u}_{j}(t)-\boldsymbol{u}_{j}\left(t^{\prime}\right)\right\|_{\boldsymbol{L}^{2}\left(\Omega, \mathbf{R}^{n}\right)}+\left\|\boldsymbol{A} \boldsymbol{u}_{j}(t)-\boldsymbol{\mathfrak { A }} \boldsymbol{u}_{j}\left(t^{\prime}\right)\right\|_{\boldsymbol{L}^{2}\left(\Omega, \mathbf{R}^{n}\right)} \\
& \leq\left\|\boldsymbol{u}_{j}(t)-\boldsymbol{u}_{j}\left(t^{\prime}\right)\right\|_{\boldsymbol{H}^{2}\left(\Omega, \mathbf{R}^{n}\right)}+\left\|\mathfrak{A} \boldsymbol{u}_{j}(t)-\mathfrak{A} \boldsymbol{u}_{j}\left(t^{\prime}\right)\right\|_{\boldsymbol{L}^{2}\left(\Omega, \mathbf{R}^{n}\right)} \\
& \leq\left(\frac{1}{C_{1}}+1\right)\left\|\boldsymbol{A} \boldsymbol{u}_{j}(t)-\mathfrak{A} \boldsymbol{u}_{j}\left(t^{\prime}\right)\right\|_{\boldsymbol{L}^{2}\left(\Omega, \mathbf{R}^{n}\right)} \\
& \longrightarrow 0
\end{aligned}
$$

This proves that $\boldsymbol{u}_{j}(t) \in C([0, \infty), D(\mathfrak{A}))$.

Summing up, we have proved that the approximate solutions $\left\{\boldsymbol{u}_{j}(t)\right\}$ enjoy the regularity property $(2.1)$ :

$$
\boldsymbol{u}_{j}(t) \in C^{2}\left([0, \infty), \boldsymbol{L}^{2}\left(\Omega, \mathbf{R}^{n}\right)\right) \cap C^{1}([0, \infty), \boldsymbol{H}) \cap C([0, \infty), D(\mathfrak{A})) .
$$

Step 3: By applying the energy inequality (2.3) to the functions

$$
\boldsymbol{u}_{j}(t)-\boldsymbol{u}_{k}(t),
$$

we obtain that

$$
\begin{aligned}
& \left\|\boldsymbol{u}_{j}(t)-\boldsymbol{u}_{k}(t)\right\|_{\boldsymbol{H}^{2}\left(\Omega, \mathbf{R}^{n}\right)}+\left\|\boldsymbol{u}_{j}^{\prime}(t)-\boldsymbol{u}_{k}^{\prime}(t)\right\|_{\mathcal{H}} \\
& +\left\|\boldsymbol{u}_{j}^{\prime \prime}(t)-\boldsymbol{u}_{k}^{\prime \prime}(t)\right\|_{\boldsymbol{L}^{2}\left(\Omega, \mathbf{R}^{n}\right)} \\
\leq & C\left(\left\|\boldsymbol{f}_{j}(0)-\boldsymbol{f}_{k}(0)\right\|_{\boldsymbol{L}^{2}\left(\Omega, \mathbf{R}^{n}\right)}+\int_{0}^{t}\left\|\boldsymbol{f}_{j}^{\prime}(s)-\boldsymbol{f}_{k}^{\prime}(s)\right\|_{\boldsymbol{L}^{2}\left(\Omega, \mathbf{R}^{n}\right)} d s\right) .
\end{aligned}
$$

However, by assertions (8.2) we have the following two assertions:

(1) $\left\{\boldsymbol{f}_{j}(0)\right\}$ is a Cauchy sequence in $\boldsymbol{L}^{2}\left(\Omega, \mathbf{R}^{n}\right)$.

(2) For any given $T>0,\left\{\boldsymbol{f}_{j}^{\prime}(s)\right\}$ is a Cauchy sequence in $\boldsymbol{L}^{2}\left(\Omega, \mathbf{R}^{n}\right)$ for each $s \in[0, T]$.

Moreover, by applying Lebesgue's dominated convergence theorem we obtain from assertion (2) that

$$
\int_{0}^{T}\left\|\boldsymbol{f}_{j}^{\prime}(s)-\boldsymbol{f}_{k}^{\prime}(s)\right\|_{\boldsymbol{L}^{2}\left(\Omega, \mathbf{R}^{n}\right)} d s \longrightarrow 0 \quad \text { as } j, k \rightarrow \infty .
$$

Hence it follows from inequality (8.11) that we have, for all $t \in[0, T]$,

$$
\begin{aligned}
& \left\|\boldsymbol{u}_{j}(t)-\boldsymbol{u}_{k}(t)\right\|_{H^{2}\left(\Omega, \mathbf{R}^{n}\right)}+\left\|\boldsymbol{u}_{j}^{\prime}(t)-\boldsymbol{u}_{k}^{\prime}(t)\right\|_{\mathcal{H}} \\
& +\left\|\boldsymbol{u}_{j}^{\prime \prime}(t)-\boldsymbol{u}_{k}^{\prime \prime}(t)\right\|_{L^{2}\left(\Omega, \mathbf{R}^{n}\right)} \\
\leq & C\left(\left\|\boldsymbol{f}_{j}(0)-\boldsymbol{f}_{k}(0)\right\|_{L^{2}\left(\Omega, \mathbf{R}^{n}\right)}+\int_{0}^{t}\left\|\boldsymbol{f}_{j}^{\prime}(s)-\boldsymbol{f}_{k}^{\prime}(s)\right\|_{\boldsymbol{L}^{2}\left(\Omega, \mathbf{R}^{n}\right)} d s\right) \\
\leq & C\left(\left\|\boldsymbol{f}_{j}(0)-\boldsymbol{f}_{k}(0)\right\|_{L^{2}\left(\Omega, \mathbf{R}^{n}\right)}+\int_{0}^{T}\left\|\boldsymbol{f}_{j}^{\prime}(s)-\boldsymbol{f}_{k}^{\prime}(s)\right\|_{\boldsymbol{L}^{2}\left(\Omega, \mathbf{R}^{n}\right)} d s\right) \\
\longrightarrow & 0 \quad \text { as } j, k \rightarrow \infty .
\end{aligned}
$$

Summing up, we have proved the following three assertions: 
(i) $\left\{\boldsymbol{u}_{j}(t)\right\}$ is a Cauchy sequence in $C\left([0, \infty), \boldsymbol{H}^{2}\left(\Omega, \mathbf{R}^{n}\right)\right)$.

(ii) $\left\{\boldsymbol{u}_{j}^{\prime}(t)\right\}$ is a Cauchy sequence in $C([0, \infty), \mathcal{H})$.

(iii) $\left\{\boldsymbol{u}_{j}^{\prime \prime}(t)\right\}$ is a Cauchy sequence in $C\left([0, \infty), \boldsymbol{L}^{2}\left(\Omega, \mathbf{R}^{n}\right)\right)$.

Therefore, by combining assertions (i), (ii) and (iii) we can find a function

$$
\boldsymbol{v}(t) \in C^{2}\left([0, \infty), \boldsymbol{L}^{2}\left(\Omega, \mathbf{R}^{n}\right)\right) \cap C^{1}([0, \infty), \boldsymbol{H}) \cap C\left([0, \infty), \boldsymbol{H}^{2}\left(\Omega, \mathbf{R}^{n}\right)\right)
$$

such that

$$
\begin{aligned}
& \boldsymbol{u}_{j}(t) \longrightarrow \boldsymbol{v}(t) \quad \text { in } C\left([0, \infty), \boldsymbol{H}^{2}\left(\Omega, \mathbf{R}^{n}\right)\right) \\
& \boldsymbol{u}_{j}^{\prime}(t) \longrightarrow \boldsymbol{v}^{\prime}(t) \quad \text { in } C([0, \infty), \boldsymbol{H}) \\
& \boldsymbol{u}_{j}^{\prime \prime}(t) \longrightarrow \boldsymbol{v}^{\prime \prime}(t) \quad \text { in } C\left([0, \infty), \boldsymbol{L}^{2}\left(\Omega, \mathbf{R}^{n}\right)\right)
\end{aligned}
$$

Step 4: Finally, by passing to the limit in the Cauchy problem

$$
\left\{\begin{array}{l}
\boldsymbol{u}_{j}^{\prime \prime}(t)=\boldsymbol{A u}_{j}+\boldsymbol{f}_{j}(t) \quad t>0, \\
\boldsymbol{u}_{j}(0)=\boldsymbol{u}_{0} \\
\boldsymbol{u}_{j}^{\prime}(0)=\boldsymbol{u}_{1}
\end{array}\right.
$$

we obtain from assertions (8.2) and (8.12) that the limit function

$$
\boldsymbol{v}(t) \in C^{2}\left([0, \infty), \boldsymbol{L}^{2}\left(\Omega, \mathbf{R}^{n}\right)\right) \cap C^{1}([0, \infty), \boldsymbol{H}) \cap C([0, \infty), D(\mathfrak{A}))
$$

is a solution of the initial-boundary value problem (5.1). Here we recall that the graph norm $\|\cdot\|_{D(\mathfrak{A})}$ is equivalent to the norm $\|\cdot\|_{\boldsymbol{H}^{2}\left(\Omega, \mathbf{R}^{n}\right)}$ (see inequalities (7.10)).

Therefore, the desired regularity property (2.1) follows from the uniqueness theorem for problem (1.1) (Theorem 6.1):

$$
\boldsymbol{u}(t)=\boldsymbol{v}(t) \in C^{2}\left([0, \infty), \boldsymbol{L}^{2}\left(\Omega, \mathbf{R}^{n}\right)\right) \cap C^{1}([0, \infty), \boldsymbol{H}) \cap C([0, \infty), D(\mathfrak{A})) .
$$

Now the proof of Theorem 8.1 is complete.

\section{Open problems}

In this last section we give two important open problems concerning the initialboundary value problem (1.1) of linear elastodynamics:

(1) The first problem is to generalize main results to the case where the domain $\Omega$ has corner singularities.

(2) The second problem is to study the case where the function $\alpha(x)$ is the characteristic function of a subset of the boundary $\partial \Omega$.

For a smooth domain $\Omega$, partial results are obtained by Hayashida [9, Theorem 1], Ibuki [12, Theorem 2], Inoue [15, Theorem B] and Ito [16, Main Theorem].

Acknowledgements The author is grateful to the referee for many valuable suggestions which improved the presentation of this paper. This research was partially supported by Grantin-Aid for General Scientific Research (No. 16340031), Ministry of Education, Culture, Sports, Science and Technology, Japan. 


\section{References}

1. R. A. Adams and J.J.F. Fournier, Sobolev spaces, second edition. Academic Press, Amsterdam Heidelberg New York Oxford (2003)

2. J. Bergh and J. Löfström, Interpolation spaces, An introduction. Springer-Verlag, Berlin New York (1976)

3. J. Chazarain et A. Piriou, Introduction à la théorie des équations aux dérivées partielles linéaires. Gauthier-Villars, Paris (1981)

4. P. G. Ciarlet, Mathematical elasticity, vol. I. Stud. Math. Appl. no. 20. North-Holland, Amsterdam New York Oxford Tokyo (1988)

5. G. Duvaut et J.-L. Lions, Les inéquations en mécanique et en physique. Dunod, Paris (1972)

6. D. Fujiwara, Concrete characterization of the domains of fractional powers of some elliptic differential operators of the second order. Proc. Japan Acad. Ser. A Math. Sci. 43, 82-86 (1967)

7. J. A. Goldstein, Semigroups and second-order differential equations. J. Func. Anal. 4, 50-70 (1969)

8. J. A. Goldstein, Semigroups of linear operators and applications. Oxford University Press, New York (1985)

9. K. Hayashida, On a mixed problem for hyperbolic equations with discontinuous boundary conditions. Publ. RIMS, Kyoto Univ. 7, 57-67 (1971/72)

10. E. Hille and R. S. Phillips, Functional analysis and semi-groups. Amer. Math. Soc. Colloq. Publ. vol. 31. Amer. Math. Soc., Providence, Rhode Island (1957)

11. L. Hörmander, The analysis of linear partial differential operators III. Grundlehren Math. Wiss. Springer-Verlag, Berlin Heidelberg New York Tokyo (1985)

12. K. Ibuki, On the regularity of solutions of a mixed problem for hyperbolic equations of second order in a domain with corners. J. Math. Kyoto Univ. 16, 167-183 (1976)

13. M. Ikawa, Mixed problems for hyperbolic equations of second order. J. Math. Soc. Japan 20, 580-608 (1968)

14. A. Inoue, On a mixed problem for $\square$ with discontinuous boundary condition (I). J. Fac. Sci. Univ. Tokyo Sect. IA 21, 85-92 (1974)

15. A. Inoue, On a mixed problem for d'Alembertian with a mixed boundary condition - an example of moving boundary. Publ. RIMS, Kyoto Univ. 11, 339-401 (1976)

16. H. Ito, On a mixed problem of linear elastodynamics with time-dependent discontinuous boundary condition. Osaka J. Math. 27, 667-707 (1990)

17. J. E. Marsden and T.J.R. Hughes, Mathematical foundations of elasticity. Prentice-Hall, Inc., Englewood Cliffs, New Jersey (1983)

18. E.M. Stein, Singular integrals and differentiability properties of functions. Princeton Math. Ser. Princeton University Press, Princeton, New Jersey (1970)

19. K. Taira, Analytic semigroups and semilinear initial-boundary value problems. London Math. Soc. Lecture Note Ser. vol. 223. Cambridge University Press, Cambridge (1995)

20. K. Taira, Introduction to boundary value problems of nonlinear elastostatics. Tsukuba J. Math. 32, 67-138 (2008)

21. K. Taira, Degenerate elliptic boundary value problems with asymmetric nonlinearity. J. Math. Soc. Japan 62, 431-465 (2010)

22. H. Triebel, Theory of function spaces. Monogr. Math. Birkhäuser Verlag, Basel (1983).

23. T. Valent, Boundary value problems of finite elasticity. Springer Tracts Nat. Phil. no 31. Springer-Verlag, New York Berlin Heidelberg Tokyo (1988)

24. B. Weiss, Abstract vibrating systems. J. Math. Mech. 17, 241-255 (1967)

25. K. Yosida, Functional analysis, sixth edition. Grundlehren Math. Wiss. Springer-Verlag, Berlin Heidelberg New York (1980) 